\title{
NONMONOTONE WAVES IN A THREE SPECIES REACTION-DIFFUSION MODEL
}

\author{
Patrick D. Miller
}

\begin{abstract}
This paper establishes the existence of a nonmonotone travelling wave for a reaction-diffusion system modeling three competing species. General existence results for travelling waves in higher dimensional systems depend on monotonicity and, therefore, do not apply to the result obtained here. The proof demonstrates an application of a homotopy invariant, the connection index, to a higher dimensional flow where few explicit results are available. The result is obtained in a singular perturbation regime where the fast-slow structure can be exploited to construct a singular limit solution from the lower dimensional reduced flows. A priori estimates show the connecting solution to be uniformly approximated by the singular limit, and these estimates make it possible to construct the higher dimensional isolating neighborhoods necessary for defining the index. The index is computed by continuing the equations to a system containing a lower dimensional invariant manifold.
\end{abstract}

\section{Introduction}

Reaction-diffusion equations are used extensively as continuous space-time models for interacting and diffusing chemical and biological species, combustion, phase transitions, and neurophysiology. In mathematical ecology, the interactions between individuals can take the form of competition for limited resources, predator-prey interactions, or mutualistic relationships where the diffusion terms model the migration of a species (see $[7,20])$. Though these equations are relatively simple, they can exhibit a variety of interesting spatial and spatio-temporal patterns, including wave fronts and wave pulses. The general system for modeling $N$ species interacting in one space dimension is of the form,

$$
\frac{\partial u_{i}}{\partial t}=d_{i} \frac{\partial^{2} u_{i}}{\partial x^{2}}+u_{i} g_{i}(u), \quad i=1, \ldots, N .
$$

Here $u_{i}(x, t)$ denotes a population density at time $t$ and spatial position $x$, and the diffusion coefficients are assumed constant, $d_{i} \geq 0$.

Travelling waves, solutions of the form $u(x, t)=U(x-\theta t)$, play an important role in the dynamics of (1.1) and are of fundamental interest. A travelling wave represents a segregated spatial pattern propagating through the spatial domain at a constant speed. A homotopy invariant, the connection index, has been successful in proving existence results for systems with two species $[5,9,12]$, and the connection matrix was used in [18] for two mutualist species in competition with a third species.

Received July 31, 1996.

1991 Mathematics Subject Classification: Primary 35K57, 34C37.

Key words and phrases: Reaction-diffusion equations, travelling waves, singular perturbation, Conley index, connection index. 
General existence results for travelling waves in $N$ species $[19,23]$ ultimately depend on monotonicity and the maximum principle, and do not extend to the results presented here. For singularly perturbed systems, geometric methods can be used to prove existence when certain transversality conditions are satisfied by the limiting solution within a lower dimensional reduced flow $[1,13,14]$.

This paper presents an existence theorem for a heteroclinic travelling wave connecting two stable rest states in a system modeling three competing species. The significance of this result is that it establishes the existence of a nonmonotone wave, where the general results for $N$ species do not apply, and the proof demonstrates a method for applying the the connection index to a system with $N>2$ where very few results have been obtained. The existence results are obtained in a singular perturbation regime where the fast-slow structure in the flow makes it possible to construct a singular limit solution from lower dimensional reduced flows. The singular limit in the fast scaling is a travelling wave for two competing species. The topological proof only requires knowing that the lower dimensional wave exists and that the wave speed is unique, as established in [5] and [8]. The higher dimensional phase space associated with the additional species increases the difficulty of showing that the index is well-defined. The fast-slow structure is used to construct the necessary isolating neighborhoods from sets which are isolating for the lower dimensional reduced flows.

For the result presented here, the governing system of reaction-diffusion equations models the evolution of three competing species:

$$
\begin{aligned}
& u_{1 t}=u_{1 x x}+u_{1} g_{1}(u), \\
& u_{2 t}=\varepsilon^{2} u_{2 x x}+r_{2} u_{2} g_{2}(u), \\
& u_{3 t}=\varepsilon^{2} d u_{3 x x}+r_{3} u_{3} g_{3}(u) .
\end{aligned}
$$

The travelling wave presented in this paper connects the two rest states $P_{2}$ and $P_{3}$, as defined by

$$
\begin{aligned}
& P_{2}: \quad g_{1}\left(u_{1}, u_{2}, 0\right)=g_{2}\left(u_{1}, u_{2}, 0\right)=0, \quad \text { with } u_{3}=0 \text {, } \\
& P_{3}: \quad g_{1}\left(u_{1}, 0, u_{3}\right)=g_{3}\left(u_{1}, 0, u_{3}\right)=0, \quad \text { with } u_{2}=0 \text {. }
\end{aligned}
$$

Travelling waves are steady state solutions in a reference frame moving along with the wave, and so, in one space dimension, they satisfy a system of ordinary differential equations. We look for slow waves with speed $\varepsilon \theta$ where $\theta(\varepsilon)$ remains $O(1)$ as $\varepsilon \rightarrow 0$. Transforming the equations to the moving frame $\zeta=x-\varepsilon \theta t$, with $U_{i}(t, \zeta)=$ $u_{i}(t, \zeta+\varepsilon \theta t)$, the travelling wave ansatz yields a flow on $\mathbb{R}^{6}$ :

$$
\begin{aligned}
\dot{U}_{1} & =V_{1}, \\
\dot{V}_{1} & =-\varepsilon \theta V_{1}-U_{1} g_{1}(U), \\
\varepsilon \dot{U}_{2} & =V_{2}, \\
\varepsilon \dot{V}_{2} & =-\theta V_{2}-r_{2} U_{2} g_{2}(U), \\
\varepsilon \dot{U}_{3} & =V_{3}, \\
\varepsilon \dot{V}_{3} & =d^{-1}\left[-\theta V_{3}-r_{3} U_{3} g_{3}(U)\right] .
\end{aligned}
$$


The singular nature of the limit $\varepsilon \rightarrow 0$ results in two distinct time scales, and the $\zeta$ parametrization is referred to throughout as the slow scaling. To introduce a fast scaling, or stretched scaling, define $\xi=\zeta / \varepsilon, u_{i}(\xi ; \varepsilon)=U_{i}(\varepsilon \xi ; \varepsilon)$, and $v_{i}(\xi ; \varepsilon)=V_{i}(\varepsilon \xi ; \varepsilon)$ :

$$
\begin{aligned}
u_{1}^{\prime} & =\varepsilon v_{1}, \\
v_{1}^{\prime} & =\varepsilon\left(-\varepsilon \theta v_{1}-u_{1} g_{1}(u)\right), \\
u_{2}^{\prime} & =v_{2}, \\
v_{2}^{\prime} & =-\theta v_{2}-r_{2} u_{2} g_{2}(u), \\
u_{3}^{\prime} & =v_{3} \\
v_{3}^{\prime} & =d^{-1}\left[-\theta v_{3}-r_{3} u_{3} g_{3}(u)\right] .
\end{aligned}
$$

For $\varepsilon>0$, equations (1.4) and (1.5) are equivalent, and their solutions will be denoted by $X(\zeta ; \varepsilon)$ and $x(\xi ; \varepsilon)$, respectively. The variables $y=\left(u_{1}, v_{1}\right)$ are referred to as the slow components and $z=\left(u_{2}, v_{2}, u_{3}, v_{3}\right)$ as the fast components. Setting $\varepsilon=0$ in (1.4) and (1.5) will be called the slow and fast reduced flows, respectively.

Proving the existence of the travelling wave for $\varepsilon>0$ means showing that there is some value of the wave speed parameter $\theta(\varepsilon)$ for which there exists a heteroclinic solution to (1.4) and (1.5) connecting the two rest points $P_{2}$ and $P_{3}$. The most difficult part of the existence proof is to find appropriate isolating neighborhoods in the six dimensional phase space such that the connection index is well-defined. Moreover, we must show that given a nontrivial index, the only possible solution is the connecting orbit which we are seeking. These properties will follow from a priori estimates showing that the solution must lie uniformly close to the singular limit solution as $\varepsilon \rightarrow 0$. The final determination of the index is made via a continuation to a product flow consisting of lower dimensional flows for which the indices are already known.

The paper continues with a statement of the hypotheses in Section 1.1. Section 2 presents the reduced equations for $\varepsilon=0$ and describes the singular limit solution. The main result is stated in Section 3 followed by an overview of the index methods employed in the proof. The remainder of Section 3 is taken up by the details of the existence proof.

1.1. Hypotheses. In the governing equations (1.2), the constants $r_{2}, r_{3}$, and $d$ are strictly positive and two of the species diffuse slowly relative to the third $(\varepsilon \ll 1)$. We assume a Lotka-Volterra competition model,

$$
g_{i}(u)=1-\sum_{j=1}^{3} \beta_{i j} u_{i}
$$

where the competition coefficients $\beta_{i j}$ are nonnegative and $\beta_{i i}=1$, for $i, j=1,2,3$. Figure 1 shows the level surfaces $g_{i}(u)=0$ and identifies the location of certain crucial intersections, denoted $P_{1}, \ldots, P_{4}$. The $u_{1}$-components of these four points will be denoted by $p_{1}, \ldots, p_{4}$. The rest points $P_{2}$ and $P_{3}$ already have been defined in (1.3) and are embedded into $\mathbb{R}^{6}$ by setting $v_{i}=0$, for $i=1,2,3$. The remaining two intersection points $P_{1}$ and $P_{4}$ lie in the planes $u_{3}=0$ and $u_{2}=0$, respectively.

The following hypotheses impose a certain geometric structure on the reduced problems, ensuring that there is an appropriate singular limit solution which will perturb 


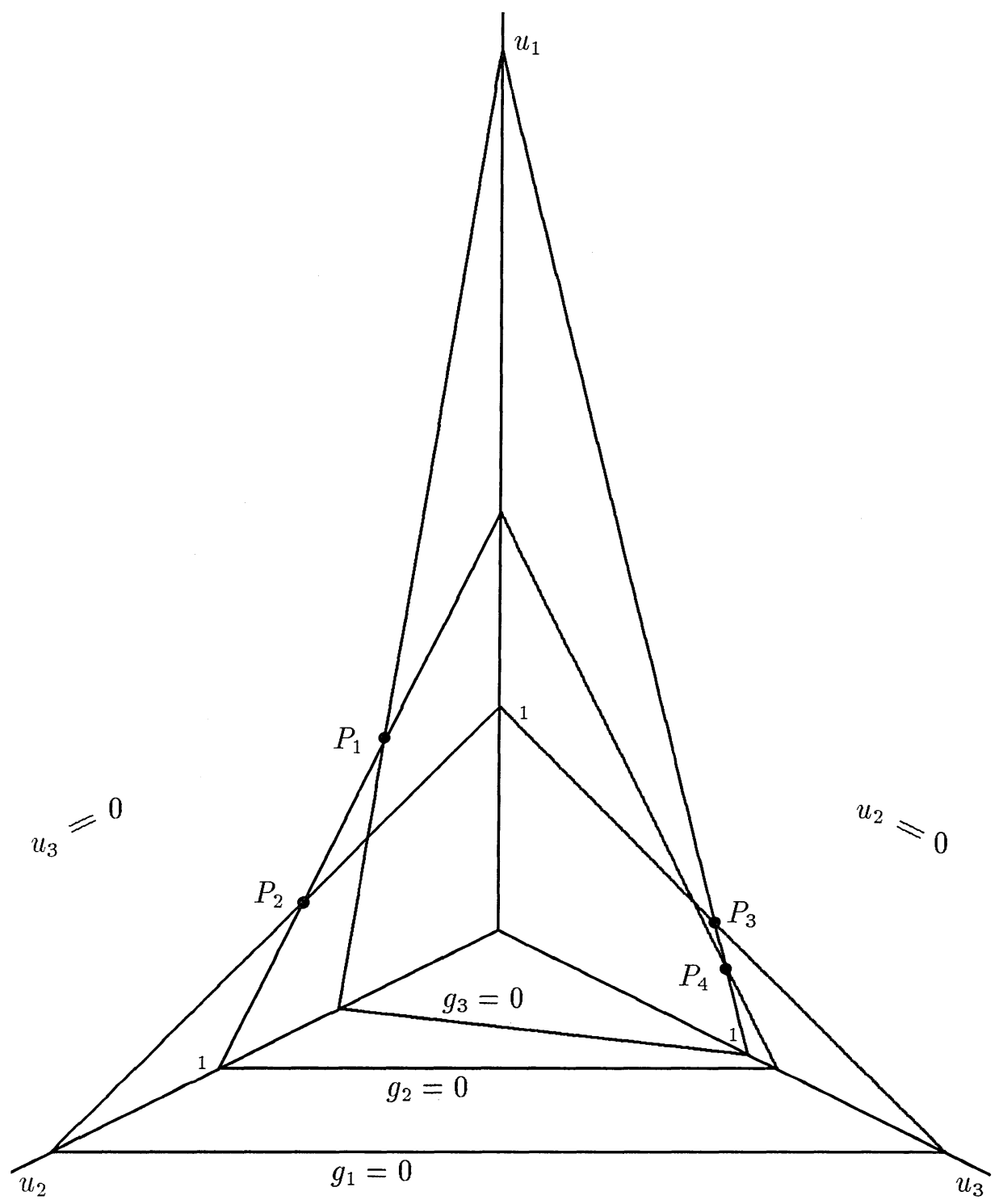

Figure 1. The zero sets of the reaction functions.

to a heteroclinic orbit for $\varepsilon>0$.

$\mathrm{H} 1: \quad 1<1 / \beta_{23}<\beta_{32}$,

H2: $\quad \beta_{12}<1$,

H3: $\quad \beta_{21}<\beta_{31}$,

$\mathrm{H} 4: \quad p_{4}<p_{3}<p_{2}<p_{1}$.

The hypotheses $p_{2}<p_{1}$ and $p_{4}<p_{3}$ ensure that $P_{2}$ and $P_{3}$ are stable rest points of the reaction flow, whereas the ordering $p_{3}<p_{2}$ is arbitrary. The motivation for 


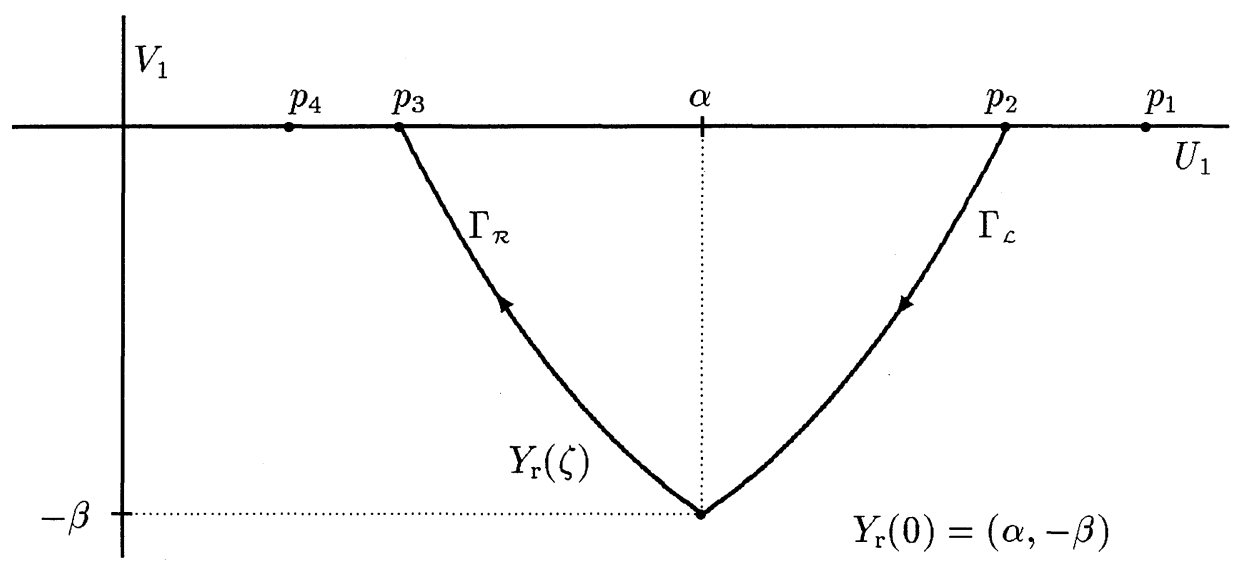

Figure 2. The slow singular limit in the $U_{1}-V_{1}$ phase space $(\varepsilon=0)$.

the remaining hypotheses will be made clearer when we define the singular limit solution. Note that these results also will hold for more general nonlinearities with an appropriate reformulation of the hypotheses in (1.7).

\section{Singular limit $(\varepsilon=0)$}

First define a singular limit solution at $\varepsilon=0$ using a singular perturbation construction (see [17]). The slow and fast scalings give distinctly different reduced flows at $\varepsilon=0$ and must be considered separately.

2.1. Slow scaling. Setting $\varepsilon=0$ in (1.4) reduces the equations for the fast components to the algebraic equation $G(Y, Z ; \theta)=0$ where $G(\cdot, \cdot ; \theta): \mathbb{R}^{6} \rightarrow \mathbb{R}^{4}$. This determines several two-dimensional manifolds, $Z=h(Y)$, with the reduced flow on the invariant slow manifold determined by setting $Z=h(Y)$ in the first two equations for $\dot{Y}$. Consider two particular pieces of the slow manifold, the left slow manifold $\mathcal{M}_{\mathcal{L}}$, determined by solving $g_{2}(u)=0$ with $u_{3}=0$, and the right slow manifold $\mathcal{M}_{\mathcal{R}}$, satisfying $g_{3}(u)=0$ with $u_{2}=0$. The rest points $P_{2}$ and $P_{3}$ lie in the left and right slow manifolds, respectively, and we denote these slow manifolds by the graphs $z=\mathcal{L}(y)=\left(\mathcal{L}_{2}\left(u_{1}\right), 0,0,0\right)$ and $z=\mathcal{R}(y)=\left(0,0, \mathcal{R}_{3}\left(u_{1}\right), 0\right)$. The reduced flows in these slow manifolds are qualitatively the same, each having a center at $(0,0)$ along with one saddle point. For the flow in $\mathcal{M}_{\mathcal{L}}$, the unstable manifold of $\left(p_{2}, 0\right)$ is denoted by the graph $V_{1}=\Gamma_{\mathcal{L}}\left(U_{1}\right)$, and for the flow in $\mathcal{M}_{\mathcal{R}}$, the stable manifold of $\left(p_{3}, 0\right)$ is the graph $V_{1}=\Gamma_{\mathcal{R}}\left(U_{1}\right)$. By their monotonicity these two curves intersect uniquely in the lower half-plane at $\left(U_{1}, V_{1}\right)=(\alpha,-\beta)$ (see Figure 2).

The slow singular limit $X_{\mathrm{r}}(\zeta)=\left(Y_{\mathrm{r}}(\zeta), Z_{\mathrm{r}}(\zeta)\right)$ satisfies $Y_{\mathrm{r}}(0)=(\alpha,-\beta)$. For $\zeta<0$, $Y_{\mathrm{r}}(\zeta)$ satisfies the reduced flow on $\mathcal{M}_{\mathcal{L}}$ with $Z_{\mathrm{r}}(\zeta)=\mathcal{L}\left(Y_{\mathrm{r}}(\zeta)\right)$, and for $\zeta>0, Y_{\mathrm{r}}(\zeta)$ satisfies the reduced flow on $\mathcal{M}_{\mathcal{R}}$ with $Z_{\mathrm{r}}(\zeta)=\mathcal{R}\left(Y_{\mathrm{r}}(\zeta)\right)$. The singular limit $X_{\mathrm{r}}(\zeta)$ is a connection from $P_{2}$ to $P_{3}$ with a discontinuous transition layer at $\zeta=0$. Graphs of the $U$-components in Figure 3 show that the slow component $U_{1}$ is continuous at $\zeta=0$, but the fast components $U_{2}$ and $U_{3}$ are discontinuous, with $U_{2}$ nonmonotone in $\zeta$. 

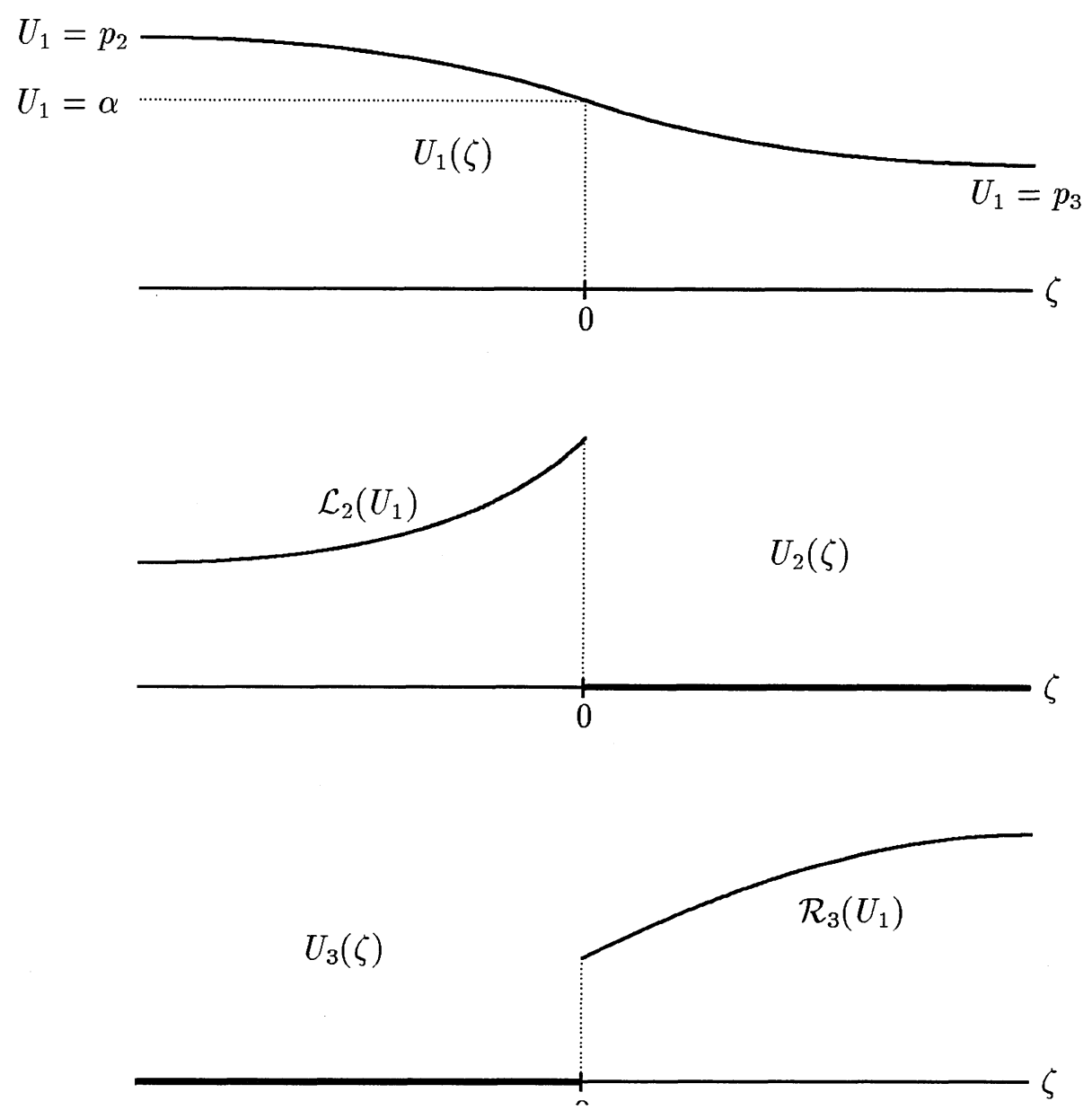

Figure 3. The singular limit in the slow scaling $(\varepsilon=0)$.

2.2. Fast scaling. The reduced flow in (1.5) is a flow on $\mathbb{R}^{4}$ parametrized by $u_{1}$. The equations are just the travelling wave equations with wave speed parameter $\theta$, as derived for the two-component reaction-diffusion system,

$$
\begin{aligned}
& u_{2 t}=u_{2 x x}+r_{2} u_{2} g_{2}(u), \\
& u_{3 t}=d u_{3 x x}+r_{3} u_{3} g_{3}(u) .
\end{aligned}
$$

The results in [5] show that for any $u_{1}$ in the interval $p_{4}<u_{1}<p_{1}$, there exists a bistable travelling wave solution to $(2.1)$ connecting $\mathcal{L}\left(u_{1}\right)$ at $\xi=-\infty$ with $\mathcal{R}\left(u_{1}\right)$ at $\xi=+\infty$. For the existence proof, we also use the result from [8] that the wave speed, denoted by $\Theta\left(u_{1}\right)$, is unique. Hypothesis $\mathrm{H} 4$ in (1.7) ensures that the transition point $u_{1}=\alpha$ lies in the interval $p_{4}<\alpha<p_{1}$. The fast singular limit $x_{\mathrm{r}}(\xi)$ is defined by this heteroclinic orbit for the fast reduced flow connecting $\mathcal{L}(\alpha)$ to $\mathcal{R}(\alpha)$ with wave speed $\Theta(\alpha)$, and $\left(u_{1}, v_{1}\right)=(\alpha,-\beta)$. The existence of this connecting orbit for the fast reduced flow at $u_{1}=\alpha$ is the mechanism by which the solution to the reduced 


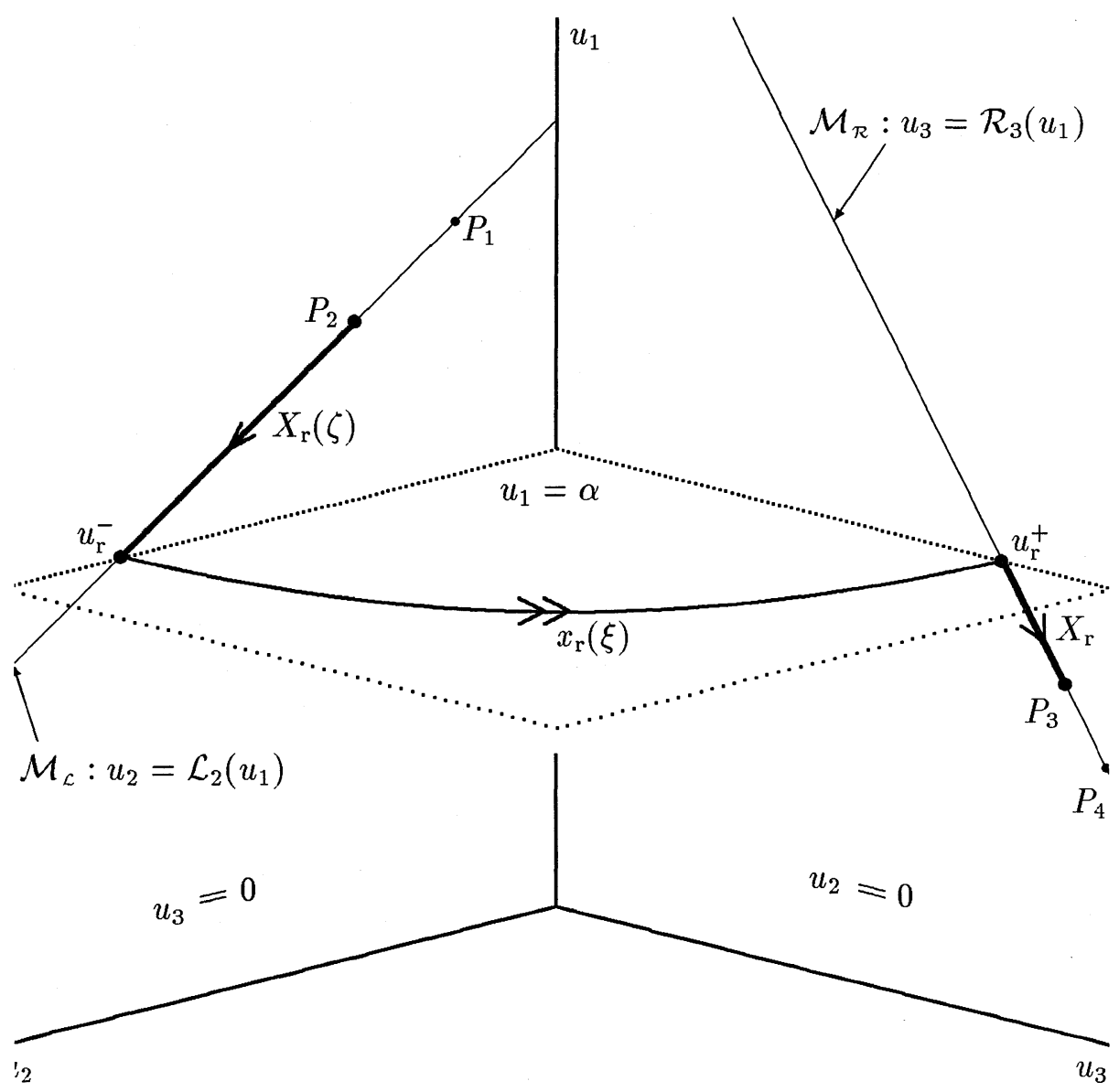

FIgURE 4. The singular limit heteroclinic solution $(\varepsilon=0)$.

equations transitions from the left slow manifold to the right slow manifold. Figure 4 shows both the fast and slow singular limits in phase space as projected onto the $u$-components.

\section{Existence theorem $(\varepsilon>0)$}

We now state the main result, that the singular limit solution described in Section 2 perturbs to a bistable heteroclinic connection for $\varepsilon>0$.

Theorem 3.1 (Existence). Suppose the hypotheses H1-H4 are satisfied. Then for all sufficiently small $\varepsilon>0$, there exists a solution $(x(\xi ; \varepsilon), \theta(\varepsilon))$ to (1.5) which connects $P_{2}$ at $\xi=-\infty$ with $P_{3}$ at $\xi=+\infty$. The wave speed $\theta(\varepsilon)$ tends to $\Theta(\alpha)$ as $\varepsilon \rightarrow 0$.

The proof uses the connection index, and we begin with an overview of these methods and some properties needed later in the proof. More complete descriptions of these methods can be found in $[3,5,9,22]$. 
3.1. Conley index. Consider a local flow on $\mathbb{R}^{n}, x^{\prime}=\varphi(x)$. For a compact set $N \subset \mathbb{R}^{n}$, let $S(N)$ denote the maximal invariant set of $N$ relative to the flow, the set of points which stay in $N$ for all time under the action of the flow. If $S(N) \cap \partial N=\emptyset$, $N$ is said to be an isolating neighborhood and $S(N)$ an isolated invariant set. $N$ is called an isolating block if the flow through each point in $\partial N$ leaves $N$ immediately in at least one time direction. The strict exit set of $N$ is the set of points in $\partial N$ which leave $N$ immediately in forward time.

The Conley index (or homotopy index) of an isolated invariant set $S$, denoted by $h(S)$, is the homotopy type $\left[N / N^{+}\right]$of an index pair $\left(N, N^{+}\right)$where $N$ is an isolating neighborhood for $S=S(N)$ and $N / N^{+}$. is the pointed space obtained by collapsing $N^{+}$to a single point. In the simplest situation, where $N$ is an isolating block, the set $N^{+}$can be taken to be the strict exit set of $N$.

A sum $(\vee)$ and product $(\Lambda)$ are defined on homotopy classes of pointed spaces and the following elementary facts are proved in [3]. If $S_{1} \subset \mathbf{X}$ and $S_{2} \subset \mathbf{X}$ are disjoint isolated invariant sets, then $h\left(S_{1} \cup S_{2}\right)=h\left(S_{1}\right) \vee h\left(S_{2}\right)$. If $S_{\mathbf{X}} \subset \mathbf{X}$ and $S_{\mathrm{Y}} \subset \mathbf{Y}$ are isolated invariant sets, then $S_{\mathrm{X}} \times S_{\mathrm{Y}}$ is an isolated invariant set in the product space $\mathbf{X} \times \mathbf{Y}$ and $h\left(S_{\mathrm{X}} \times S_{\mathrm{Y}}\right)=h\left(S_{\mathrm{X}}\right) \wedge h\left(S_{\mathrm{Y}}\right)$. The additive identity is the homotopy class of the empty set, the pointed one-point space denoted by $\overline{0}$. The multiplicative identity is the homotopy class of an isolated attracting rest point, the pointed two-point space or pointed zero-sphere denoted by $\overline{1}$. An isolated hyperbolic rest point with $k$ unstable eigenvalues (Morse index $k$ ) has the homotopy class of the pointed $k$-sphere denoted by $\Sigma^{k}$.

3.2. Connection index. The connection index extends the Conley index to the existence of heteroclinic solutions to parametrized flows, $x^{\prime}=\varphi(x ; \theta)$ where $x \in \mathbb{R}^{n}$, and $\theta \in \mathbb{R}$. The flow is augmented with the parameter equation $\theta^{\prime}=0$, and for $S$ an invariant set relative to the augmented flow on $\mathbb{R}^{n+1}$, let $S_{\theta} \subset \mathbb{R}^{n}$ denote the subset of $S$ with parameter value $\theta$.

Definition. Suppose $\left(S^{\prime}, S^{\prime \prime}, S\right)$ is a triple of invariant sets relative to the augmented flow on $\mathbb{R}^{n} \times\left[\theta_{1}, \theta_{2}\right]$. Then $\left(S^{\prime}, S^{\prime \prime}, S\right)$ is called a connection triple if $S_{\theta}^{\prime}$, $S_{\theta}^{\prime \prime}$, and $S_{\theta}$ are isolated invariant sets for each $\theta \in\left[\theta_{1}, \theta_{2}\right]$ and the triple satisfies

1. $S^{\prime} \cup S^{\prime \prime} \subset S$,

2. $S^{\prime} \cap S^{\prime \prime}=\emptyset$,

3. $S_{\theta}=S_{\theta}^{\prime} \cup S_{\theta}^{\prime \prime} \quad$ for $\theta=\theta_{1}, \theta_{2}$.

The connection index for a connection triple $\left(S^{\prime}, S^{\prime \prime}, S\right)$ is rigorously defined via the Conley index applied to an appropriately perturbed flow $[4,5]$. In many applications $S^{\prime}$ and $S^{\prime \prime}$ are just curves of rest points, and we seek a connecting orbit from $S_{\theta}^{\prime}$ to $S_{\theta}^{\prime \prime}$ for some value of the parameter $\theta$. To visualize the calculation of the connection index, suppose $\left(N, N^{+}\right)$is an index pair for the isolated invariant set $S_{\theta}$ for all $\theta \in\left[\theta_{1}, \theta_{2}\right]$. For $S_{\theta}^{\prime} \subset N$, define $\mathcal{W}^{\mathcal{U}}\left(S_{\theta}^{\prime}, N\right)$ as the set of all points in $N$ which tend to $S_{\theta}^{\prime}$ in backwards time, the unstable manifold of $S_{\theta}^{\prime}$. In particular, define $\mathcal{W}_{1}^{\mathcal{U}}=\mathcal{W}^{\mathcal{U}}\left(S_{\theta_{1}}^{\prime}, N\right)$ and $\mathcal{W}_{2}^{\mathcal{U}}=\mathcal{W}^{\mathcal{U}}\left(S_{\theta_{2}}^{\prime}, N\right)$, and define the compact sets in $\mathbb{R}^{n} \times\left[\theta_{1}, \theta_{2}\right]$,

$$
\begin{aligned}
\bar{N} & =N \times\left[\theta_{1}, \theta_{2}\right] \\
\bar{N}^{+} & =\left(N^{+} \times\left[\theta_{1}, \theta_{2}\right]\right) \cup \mathcal{W}_{1}^{\mathcal{U}} \cup \mathcal{W}_{2}^{\mathcal{U}} .
\end{aligned}
$$


Then the connection index is defined heuristically as $\bar{h}\left(S^{\prime}, S^{\prime \prime}, S\right)=\left[\bar{N} / \bar{N}^{+}\right]$. The existence of a connecting orbit follows from the result

$$
\bar{h}\left(S^{\prime}, S^{\prime \prime}, S\right) \neq\left(\Sigma^{1} \wedge h\left(S^{\prime}\right)\right) \vee h\left(S^{\prime \prime}\right) \rightarrow S \neq S^{\prime} \cup S^{\prime \prime}
$$

where $\Sigma^{1}$ is the pointed one-sphere and $h\left(S^{\prime}\right)$ and $h\left(S^{\prime \prime}\right)$ are just $h\left(S_{\theta}^{\prime}\right)$ and $h\left(S_{\theta}^{\prime \prime}\right)$ for any $\theta \in\left[\theta_{1}, \theta_{2}\right]$. If the computed index satisfies the inequality on the left-hand side of (3.1), additional knowledge of $S$ is needed to conclude that $S \backslash\left(S^{\prime} \cup S^{\prime \prime}\right)$ contains a connecting solution between $S^{\prime}$ and $S^{\prime \prime}$.

Now suppose that the flow $x^{\prime}=\varphi(x ; \theta ; \mu)$ is parametrized continuously by $\mu \in$ $[0,1]$. Let $N_{\mu}^{\prime}, N_{\mu}^{\prime \prime}$, and $N_{\mu}$ be isolating neighborhoods in $\mathbb{R}^{n}$ depending continuously on $\mu$ and determining a connection triple $\left(S_{\mu}^{\prime}, S_{\mu}^{\prime \prime}, S_{\mu}\right)$ on $\mathbb{R}^{n} \times\left[\theta_{1}, \theta_{2}\right]$, for all $\mu \in$ $[0,1]$. Then the triples $\left(S_{0}^{\prime}, S_{0}^{\prime \prime}, S_{0}\right)$ and $\left(S_{1}^{\prime}, S_{1}^{\prime \prime}, S_{1}\right)$ are related by continuation, and $\bar{h}\left(S_{0}^{\prime}, S_{0}^{\prime \prime}, S_{0}\right)=\bar{h}\left(S_{1}^{\prime}, S_{1}^{\prime \prime}, S_{1}\right)$. In our application, the index will be computed by continuing the equations to a product flow,

$$
\begin{aligned}
& x^{\prime}=A x \quad x \in \mathbb{R}^{p}, \\
& y^{\prime}=g(y ; \theta) \quad y \in \mathbb{R}^{q}, \quad \theta \in \mathbb{R}
\end{aligned}
$$

where $A$ is a hyperbolic constant matrix with $k$ unstable eigenvalues. If $\left(\tilde{S}^{\prime}, \tilde{S}^{\prime \prime}, \tilde{S}\right)$ is a connection triple for the flow on $\mathbb{R}^{q} \times\left[\theta_{1}, \theta_{2}\right]$ and $\left(S^{\prime}, S^{\prime \prime}, S\right)$ is the inclusion of $\left(\tilde{S}^{\prime}, \tilde{S}^{\prime \prime}, \tilde{S}\right)$ into $\mathbb{R}^{p+q}$ with the $x$-coordinates set to zero, then $\left(S^{\prime}, S^{\prime \prime}, S\right)$ is a connection triple for the product flow on $\mathbb{R}^{p+q} \times\left[\theta_{1}, \theta_{2}\right]$ with index

$$
\bar{h}\left(S^{\prime}, S^{\prime \prime}, S\right)=\Sigma^{k} \wedge \bar{h}\left(\tilde{S}^{\prime}, \tilde{S}^{\prime \prime}, \tilde{S}\right) .
$$

3.3. Continuing the equations. We first describe the deformation of the equations to be used later in computing the index. The continuation is done in three steps. (1) $\beta_{32}$ increases to a value $\bar{\beta}_{32}$ sufficiently large to make $p_{1}>1$. (2) $\beta_{12}$ goes to 0 making $p_{2}=1$. (3) $\beta_{13}$ goes to 0 making $p_{3}=1$. It is clear that the hypotheses H1-H4 remain satisfied throughout the homotopy.

Notation. To simplify the notation, we use the parameter $\mu \in[0,1]$ to denote the deformation of the flow where $\mu=1$ refers to the original equations and $\mu=0$ denotes the flow with $\beta_{32}=\bar{\beta}_{32}, \beta_{12}=0$, and $\beta_{13}=0$.

At $\mu=0$, the $g_{1}=0$ surface coincides with $u_{1}=1$, so that $p_{3}=p_{2}=1$, and the fast components have been decoupled from the flow for $\left(u_{1}, v_{1}\right)$ :

$$
\begin{aligned}
u_{1}^{\prime} & =\varepsilon v_{1}, \\
v_{1}^{\prime} & =\varepsilon\left(-\varepsilon \theta v_{1}-u_{1}\left(1-u_{1}\right)\right), \\
u_{2}^{\prime} & =v_{2}, \\
v_{2}^{\prime} & =-\theta v_{2}-r_{2} u_{2} g_{2}\left(u_{1}, u_{2}, u_{3}\right), \\
u_{3}^{\prime} & =v_{3} \\
v_{3}^{\prime} & =d^{-1}\left[-\theta v_{3}-r_{3} u_{3} g_{3}\left(u_{1}, u_{2}, u_{3}\right)\right] .
\end{aligned}
$$

The four-dimensional manifold $\mathcal{M}_{0}=\left\{x \in \mathbb{R}^{6}: u_{1}=1, v_{1}=0\right\}$ contains the rest points $P_{2}$ and $P_{3}$ and is invariant under the flow in (3.4). The flow in $\mathcal{M}_{0}$ is governed by the fast reduced flow with $u_{1}=1$, and a connection exists from $P_{2}$ with $P_{3}$. In Section 3.8, the system in (3.4) is continued to the product of a hyperbolic linear flow on $\mathbb{R}^{2}$ and a parametrized flow on $\mathbb{R}^{4}$ where we then can apply the result in (3.3). 
3.4. The connection triple. In this section, we define a connection triple relative to the flow in (1.4) and (1.5) with $\varepsilon>0$ and for all $\mu \in[0,1]$. First choose $\theta_{1}$ and $\theta_{2}$ such that $\Theta(\alpha) \in\left(\theta_{1}, \theta_{2}\right)$. Using the uniqueness of the wave speed for the fast singular limit as established in [8], it is shown in Lemma 3.6 that $\theta(\varepsilon)$ converges to $\Theta(\alpha)$ as $\varepsilon \rightarrow 0$. It follows that for $\varepsilon$ sufficiently small, a connection from $P_{2}$ to $P_{3}$ cannot exist at $\theta=\theta_{1}$ and $\theta=\theta_{2}$.

The goal is to define an appropriate neighborhood $N \subset \mathbb{R}^{6}$ for which the total invariant set consists only of connections from $P_{2}$ to $P_{3}$, and which is isolating relative to (1.4) and (1.5) for all $\theta \in\left[\theta_{1}, \theta_{2}\right]$. The invariant sets in $\mathbb{R}^{6} \times\left[\theta_{1}, \theta_{2}\right]$ which make up the triple $\left(S^{\prime}, S^{\prime \prime}, S\right)$ are defined by $S_{\theta}^{\prime}=P_{2}, S_{\theta}^{\prime \prime}=P_{3}$, and $S_{\theta}=S_{\theta}(N ; \varepsilon)$. We construct two neighborhoods, $N_{0}(\delta)$ and $N_{1}(\delta)$, with $N_{0}$ isolating for $\mu \in[0, \bar{\mu}]$ and $N_{1}$ isolating for $\mu \in[\bar{\mu}, 1]$ where $\bar{\mu}$ is to be specified later. Here $\delta=\left(\delta_{\mathrm{f}}, \delta_{0}, \delta_{1}, \delta_{2}, \delta_{3}\right)$ is a vector of small parameters used in defining the neighborhoods, and a key feature of the construction is that these parameters must be set in relation to one another, with

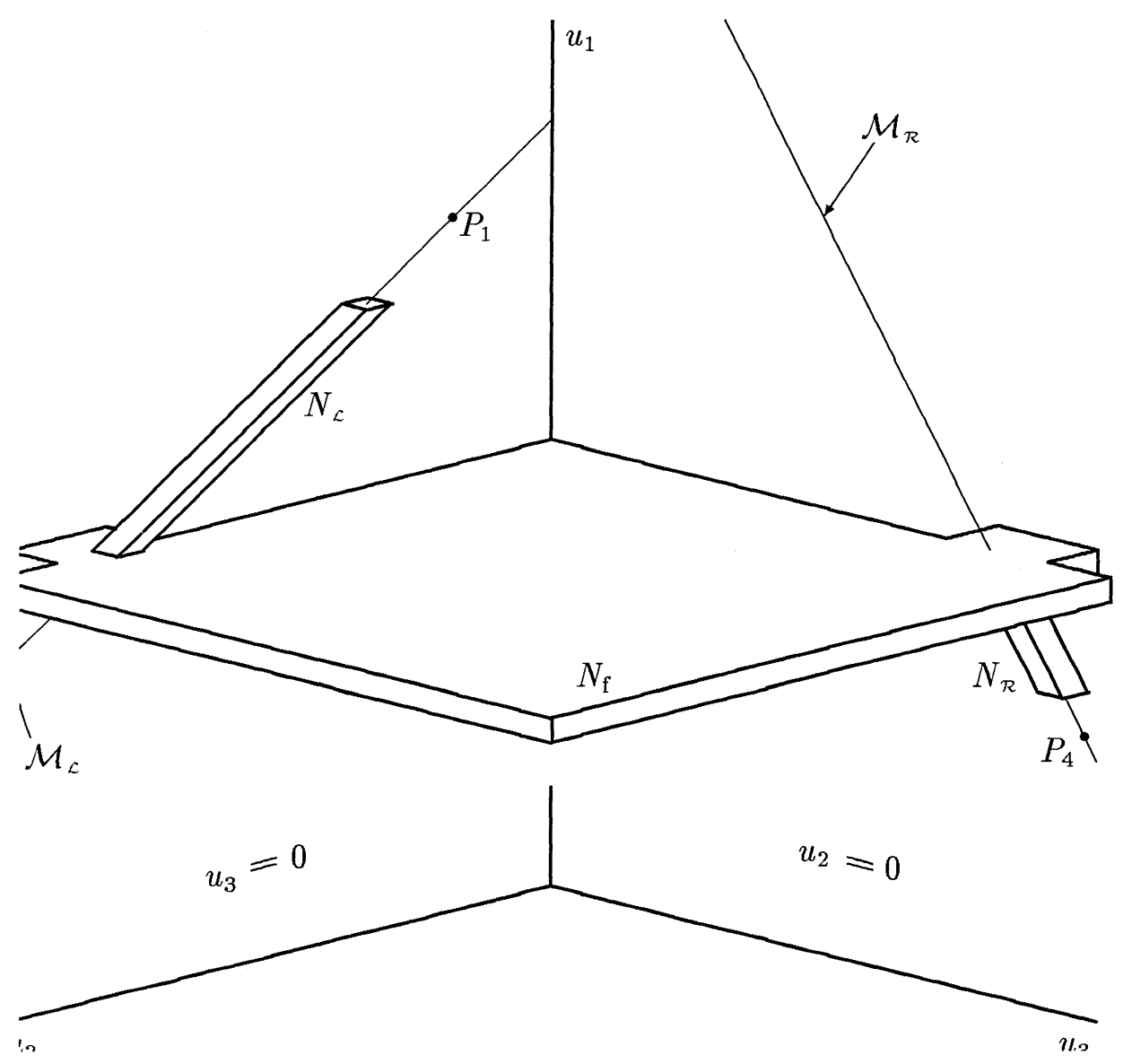

FiguRE 5. The isolating neighborhood $N_{1}$. 
the order prescribed as follows:

$$
\text { fix } \delta_{\mathrm{f}} \rightarrow \text { fix } \delta_{1} \rightarrow \text { fix } \bar{\mu} \rightarrow \text { fix } \delta_{1} \rightarrow \text { fix } \delta_{2} \rightarrow \text { fix } \delta_{3} .
$$

The critical construction is the neighborhood $N_{1}$, which consists of three pieces, a fast block $N_{\mathrm{f}}$ containing the fast singular limit, and two slow tubes, $N_{\mathcal{L}}$ and $N_{\mathcal{R}}$, containing the slow singular limit for $\zeta<0$ and $\zeta>0$, respectively. The projection of $N_{1}$ onto the three-dimensional $u$-space is shown in Figure 5 .

Notation. In describing the isolating neighborhoods, $N$ is used to denote neighborhoods in $\mathbb{R}^{6}, \mathcal{U}$ denotes sets in $\left(u_{2}, u_{3}\right)$ space, $\mathcal{V}$ denotes sets in $\left(v_{2}, v_{3}\right)$ space, $\mathcal{Z}$ denotes sets in $\mathbb{R}^{4}(\mathcal{Z}=\mathcal{U} \times \mathcal{V})$, and $\mathcal{Y}$ denotes sets in $\left(u_{1}, v_{1}\right)$ space. $S(N ; \varepsilon)$ denotes the maximal invariant set in $N$ relative to the flow in (1.4) and (1.5), parametrized by $\theta$ and $\varepsilon$. The notation $S_{\mu}(N ; \varepsilon)$ is used whenever it is necessary to be explicit about the value of the homotopy parameter, with $S_{1}(N ; \varepsilon)$ denoting the original flow in (1.5) and $S_{0}(N ; \varepsilon)$ denoting the flow at the end of the homotopy in (3.4). The notation $S_{\mathrm{f}}\left(\mathcal{Z} ; u_{1}\right)$ represents the maximal invariant set of $\mathcal{Z} \subset \mathbb{R}^{4}$ relative to the fast reduced flow parametrized by $u_{1}$.

3.4.1. Isolating neighborhoods for $P_{2}$ and $P_{3}$. It is easy to verify that for all sufficiently small $\varepsilon>0, P_{2}$ and $P_{3}$ are hyperbolic rest points of the flow in (1.5), each having three stable and three unstable eigenvalues. From singular perturbation theory [6], the slow manifold perturbs to an invariant manifold for $\varepsilon>0$, and, by the hyperbolicity of this manifold, any solution which remains in a small neighborhood of $P_{2}$ (resp. $P_{3}$ ) for all time must lie in this invariant manifold. Since the flow on the invariant manifold is an $O(\varepsilon)$ perturbation of the flow on the slow manifold for the reduced equations, it follows that for all sufficiently small neighborhoods $N^{\prime}$ and $N^{\prime \prime}$ and $\varepsilon>0, S\left(N^{\prime} ; \varepsilon\right)=P_{2}$ and $S\left(N^{\prime \prime} ; \varepsilon\right)=P_{3}$. Moreover, the isolating properties of $N^{\prime}$ and $N^{\prime \prime}$ do not depend on the smallness of $v_{2}$ and $v_{3}$, which follows from $v_{2}$ and $v_{3}$ being the derivatives of $u_{2}$ and $u_{3}$. We summarize in the following lemma.

Lemma 3.2. Suppose $N^{\prime} \subset \mathbb{R}^{6}$ is a compact neighborhood of $P_{2}$ satisfying

$$
\left|u_{1}-p_{2}\right|,\left|v_{1}\right|,\left|u_{2}-\mathcal{L}_{2}\left(p_{2}\right)\right|,\left|u_{3}\right| \leq \delta_{c}, \quad\left|v_{2}\right|,\left|v_{3}\right| \leq K,
$$

and $N^{\prime \prime}$ is a neighborhood of $P_{3}$ satisfying

$$
\left|u_{1}-p_{3}\right|,\left|v_{1}\right|,\left|u_{3}-\mathcal{R}_{3}\left(p_{3}\right)\right|,\left|u_{2}\right| \leq \delta_{c}, \quad\left|v_{2}\right|,\left|v_{3}\right| \leq K .
$$

Then for all $\delta_{c}$ and $\varepsilon$ sufficiently small, $S\left(N^{\prime} ; \varepsilon\right)=P_{2}$ and $S\left(N^{\prime \prime} ; \varepsilon\right)=P_{3}$.

3.4.2. The fast block. The fast block is given by $N_{\mathrm{f}}=\mathcal{Y}_{\mathrm{f}} \times \mathcal{Z}_{\mathrm{f}}$ where $\mathcal{Z}_{\mathrm{f}}$ is isolating relative to the fast reduced flow with $u_{1}=\alpha$, and $\mathcal{Y}_{\mathrm{f}}$ is a rectangle in $\left(u_{1}, v_{1}\right)$ space centered at the transition point $(\alpha,-\beta)$,

$$
\mathcal{Y}_{\mathrm{f}}=\mathcal{Y}_{\mathrm{f}}\left(\delta_{1}\right)=\left\{\left(u_{1}, v_{1}\right):\left|u_{1}-\alpha\right| \leq \delta_{1},\left|v_{1}+\beta\right| \leq b\right\} .
$$

For $K$ fixed and assumed large, define the sets

$$
\begin{aligned}
\mathcal{U}_{K} & =\left\{\left(u_{2}, u_{3}\right): 0 \leq u_{2}, u_{3} \leq K\right\}, \\
\mathcal{V}_{K} & =\left\{\left(v_{2}, v_{3}\right): 0 \leq-v_{2}, v_{3} \leq K\right\}, \\
\mathcal{V}_{\mathrm{f}} & =\left\{\left(v_{2}, v_{3}\right):\left|v_{2}\right|,\left|v_{3}\right| \leq K\right\} .
\end{aligned}
$$

Define the sets $\mathcal{U}_{\mathrm{f} \mathcal{L}}$ and $\mathcal{U}_{\mathrm{f} \mathcal{R}}$ to be rectangles in $\left(u_{2}, u_{3}\right)$ space centered at the slow manifolds with $u_{1}=\alpha$ (see Figure 6).

$$
\mathcal{U}_{\mathrm{f} \mathcal{L}}\left(\delta_{\mathrm{f}}\right)=\left\{\left(u_{2}, u_{3}\right):\left|u_{2}-\mathcal{L}_{2}(\alpha)\right| \leq a_{\mathcal{L}} \delta_{\mathrm{f}} ;\left|u_{3}\right| \leq \delta_{\mathrm{f}}\right\}
$$




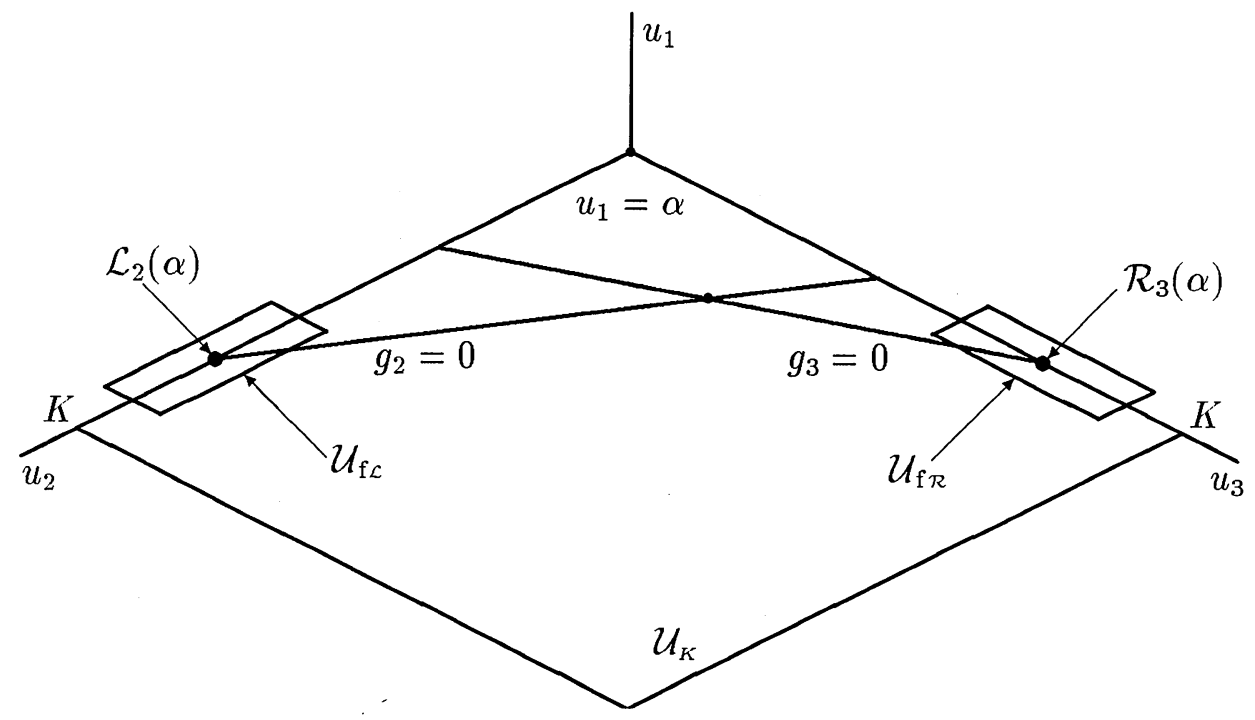

FIGURE $6 . \mathcal{U}_{\mathcal{K}}, \mathcal{U}_{\mathrm{f} \mathcal{L}}, \mathcal{U}_{\mathrm{f} \mathcal{R}}$ : isolating neighborhood for the fast components.

$$
\mathcal{U}_{\mathrm{f} \mathcal{R}}\left(\delta_{\mathrm{f}}\right)=\left\{\left(u_{2}, u_{3}\right):\left|u_{2}\right| \leq \delta_{\mathrm{f}} ;\left|u_{3}-\mathcal{R}_{3}(\alpha)\right| \leq a_{\mathcal{R}} \delta_{\mathrm{f}}\right\} .
$$

Here the positive constants $a_{\mathcal{L}}$ and $a_{\mathcal{R}}$ denote the aspect ratios of the respective rectangles, $\delta_{\mathrm{f}}$ controls the size of the neighborhoods, and we assume that $\max \left\{\delta_{\mathrm{f}}, a_{\mathcal{L}} \delta_{\mathrm{f}}, a_{\mathcal{R}} \delta_{\mathrm{f}}\right\}$ is less than $\delta_{c}$ as defined in Lemma 3.2. Now define the following sets in $\mathbb{R}^{4}, \mathcal{Z}_{\mathcal{K}}=$ $\mathcal{U}_{\mathcal{K}} \times \mathcal{V}_{\mathcal{K}}, \mathcal{Z}_{\mathrm{f} \mathcal{L}}\left(\delta_{\mathrm{f}}\right)=\mathcal{U}_{\mathrm{f} \mathcal{L}}\left(\delta_{\mathrm{f}}\right) \times \mathcal{V}_{\mathrm{f}}$, and $\mathcal{Z}_{\mathrm{fR}}\left(\delta_{\mathrm{f}}\right)=\mathcal{U}_{\mathrm{fR}}\left(\delta_{\mathrm{f}}\right) \times \mathcal{V}_{\mathrm{f}}$. Excise neighborhoods $\mathcal{Z}^{\prime}$ and $\mathcal{Z}^{\prime \prime}$ about the rest point at the origin in $\mathbb{R}^{4}$ and the rest point at $g_{2}=g_{3}=0$. The fast block in $\mathbb{R}^{4}$ is defined as

$$
\mathcal{Z}_{\mathrm{f}}=\mathcal{Z}_{\mathrm{f}}\left(\delta_{\mathrm{f}}\right)=\left(\mathcal{Z}_{\mathrm{f} \mathcal{L}}\left(\delta_{\mathrm{f}}\right) \cup \mathcal{Z}_{\mathcal{K}} \cup \mathcal{Z}_{\mathrm{fR}}\left(\delta_{\mathrm{f}}\right)\right) \backslash\left(\mathcal{Z}^{\prime} \cup \mathcal{Z}^{\prime \prime}\right)
$$

The following lemma is proved by Conley and Gardner in [5] where it is shown that the travelling wave exists for the fast reduced system.

Lemma 3.3. There exists $a_{\mathcal{L}}, a_{\mathcal{R}}>0$ such that for all $K$ sufficiently large and all $\mathcal{Z}^{\prime}$, $\mathcal{Z}^{\prime \prime}, \delta_{f}$ sufficiently small, $\mathcal{Z}_{f \mathcal{L}}\left(\delta_{f}\right)$ isolates the rest point $\mathcal{L}(\alpha), \mathcal{Z}_{f \mathcal{R}}\left(\delta_{f}\right)$ isolates the rest point $\mathcal{R}(\alpha)$, and $\mathcal{Z}_{f}\left(\delta_{f}\right)$ is an isolating neighborhood in $\mathcal{R}^{4}$, relative to the fast reduced system with $u_{1}=\alpha$. By the monotonicity built into $\mathcal{Z}_{\mathcal{K}}$, it follows that $S_{f}\left(\mathcal{Z}_{f} ; \alpha\right)$ consists only of connections from $\mathcal{L}(\alpha)$ to $\mathcal{R}(\alpha)$.

3.4.3. The slow tubes. First define tubes in $\left(u_{1}, v_{1}\right)$ space, $\tilde{\mathcal{Y}}_{\mathcal{L}}$ and $\tilde{\mathcal{Y}}_{\mathcal{R}}$, which extend beyond the slow singular limit, as shown in Figure 7:

$$
\begin{aligned}
& \tilde{\mathcal{Y}}_{\mathcal{L}}\left(\delta_{2}\right)=\bigcup_{u_{1} \in\left[p_{4}, p_{1}\right]}\left\{u_{1}\right\} \times \mathcal{I}_{\mathcal{L}}\left(\delta_{2} ; u_{1}\right), \\
& \tilde{\mathcal{Y}}_{\mathcal{R}}\left(\delta_{2}\right)=\bigcup_{u_{1} \in\left[p_{4}, p_{1}\right]}\left\{u_{1}\right\} \times \mathcal{I}_{\mathcal{R}}\left(\delta_{2} ; u_{1}\right) .
\end{aligned}
$$

Here $\mathcal{I}_{\mathcal{L}}\left(\delta_{2} ; u_{1}\right)$ denotes an interval in $v_{1}$ of length $2 \delta_{2}$ centered at the point $\left(u_{1}, \Gamma_{\mathcal{L}}\left(u_{1}\right)\right)$ on the unstable manifold of $\left(p_{2}, 0\right)$, and $\mathcal{I}_{\mathcal{R}}$ is an interval centered at $\left(u_{1}, \Gamma_{\mathcal{R}}\left(u_{1}\right)\right)$ on the stable manifold of $\left(p_{3}, 0\right)$. 


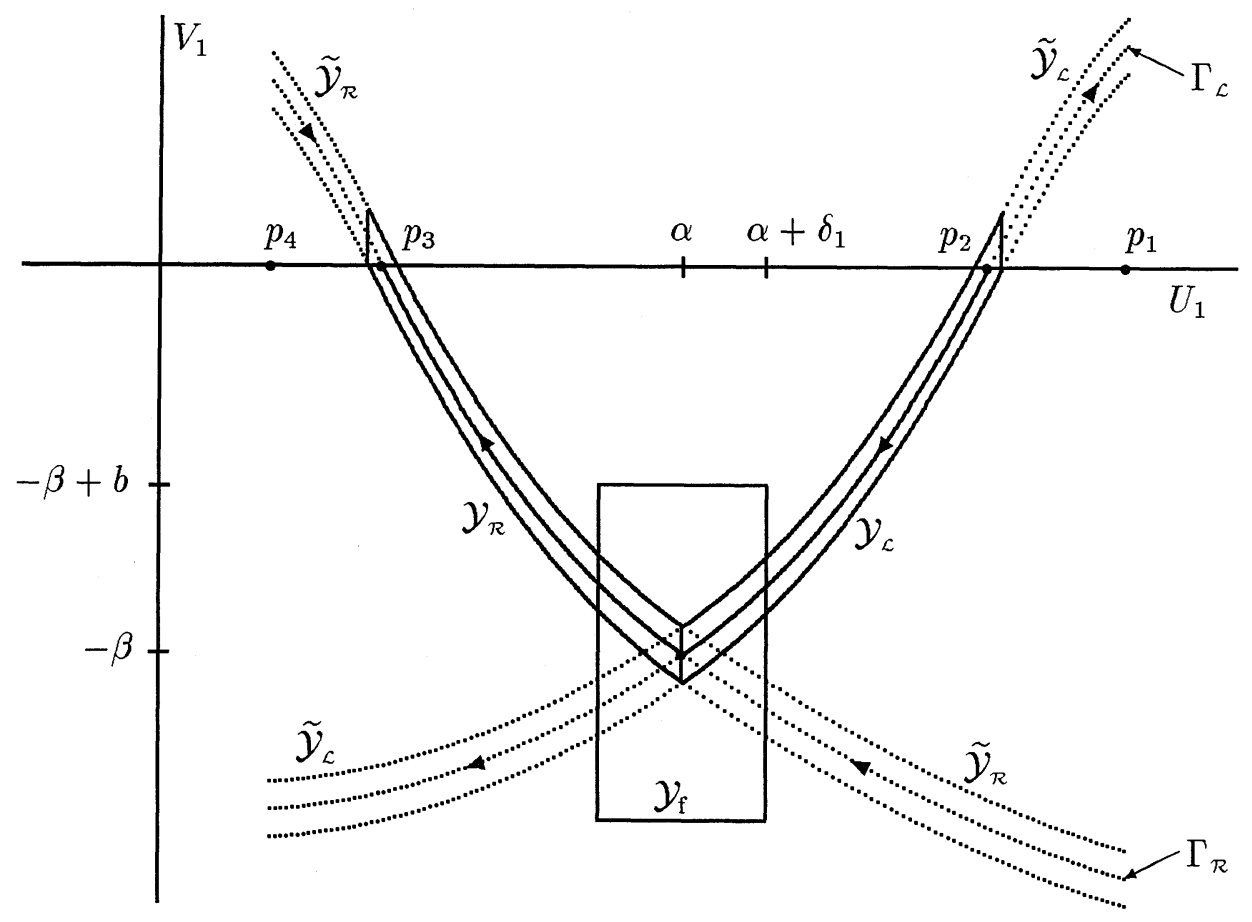

FIGURE $7 . \mathcal{Y}_{\mathrm{f}}, \mathcal{Y}_{\mathcal{L}}, \mathcal{Y}_{\mathcal{R}}$ : isolating neighborhoods for the slow components.

For each fixed $u_{1}$, define the sets in $\mathbb{R}^{4}, \mathcal{Z}_{\mathcal{L}}=\mathcal{U}_{\mathcal{L}} \times \mathcal{V}_{\mathrm{s}}$ and $\mathcal{Z}_{\mathcal{R}}=\mathcal{U}_{\mathcal{R}} \times \mathcal{V}_{\mathrm{s}}$, such that the set $\left\{u_{1}\right\} \times \mathcal{I}_{\mathcal{L}} \times \mathcal{Z}_{\mathcal{L}}$ is a neighborhood in $\mathbb{R}^{5}$ centered on the slow singular limit in $\mathcal{M}_{\mathcal{L}}$, and $\left\{u_{1}\right\} \times \mathcal{I}_{\mathcal{R}} \times \mathcal{Z}_{\mathcal{R}}$ is a neighborhood centered on the slow singular limit in $\mathcal{M}_{\mathcal{R}}$. Here $\mathcal{V}_{\mathrm{s}}\left(\delta_{3}\right)$ is a square in $\left(v_{2}, v_{3}\right)$ space centered at the origin with sides of length $2 \delta_{3}$, and $\mathcal{U}_{\mathcal{L}}$ and $\mathcal{U}_{\mathcal{R}}$ are defined by

$$
\begin{gathered}
\mathcal{U}_{\mathcal{L}}=\mathcal{U}_{\mathcal{L}}\left(\delta_{3} ; u_{1}\right)=\left\{\left(u_{2}, u_{3}\right):\left|u_{2}-\mathcal{L}_{2}\left(u_{1}\right)\right| \leq a_{\mathcal{L}} \delta_{3} ;\left|u_{3}\right| \leq \delta_{3}\right\}, \\
\mathcal{U}_{\mathcal{R}}=\mathcal{U}_{\mathcal{R}}\left(\delta_{3} ; u_{1}\right)=\left\{\left(u_{2}, u_{3}\right):\left|u_{2}\right| \leq \delta_{3} ;\left|u_{3}-\mathcal{R}_{3}\left(u_{1}\right)\right| \leq a_{\mathcal{R}} \delta_{3}\right\} .
\end{gathered}
$$

The parameter $\delta_{3}$ is chosen small relative to the parameter $\delta_{\mathrm{f}}$, so that $\mathcal{U}_{\mathcal{L}}\left(\delta_{3} ; u_{1}\right)$ lies interior to $\mathcal{U}_{\mathrm{f} \mathcal{L}}\left(\delta_{\mathrm{f}}\right)$, and $\mathcal{U}_{\mathcal{R}}$ lies interior to $\mathcal{U}_{\mathrm{f} \mathcal{R}}$.

Define two slow tubes in $\mathbb{R}^{6}$ containing the slow singular limit by letting $u_{1}$ range over an appropriately chosen interval:

$$
\begin{aligned}
& N_{\mathcal{L}}=N_{\mathcal{L}}(\delta)=\bigcup_{u_{1} \in\left[\alpha, \sigma_{\mathcal{L}}\right]}\left\{u_{1}\right\} \times \mathcal{I}_{\mathcal{L}}\left(\delta_{2} ; u_{1}\right) \times \mathcal{Z}_{\mathcal{L}}\left(\delta_{3} ; u_{1}\right), \\
& N_{\mathcal{R}}=N_{\mathcal{R}}(\delta)=\bigcup_{u_{1} \in\left[\sigma_{\mathcal{R}}, \alpha\right]}\left\{u_{1}\right\} \times \mathcal{I}_{\mathcal{R}}\left(\delta_{2} ; u_{1}\right) \times \mathcal{Z}_{\mathcal{R}}\left(\delta_{3} ; u_{1}\right)
\end{aligned}
$$

where $\sigma_{\mathcal{L}}$ and $\sigma_{\mathcal{R}}$ are defined in Figure 8. The projection of these tubes in $\left(u_{1}, v_{1}\right)$ space are denoted by $\mathcal{Y}_{\mathcal{L}}\left(\delta_{2}\right)$ and $\mathcal{Y}_{\mathcal{R}}\left(\delta_{2}\right)$, respectively, with $\mathcal{Y}_{\mathcal{L}} \subset \tilde{\mathcal{Y}}_{\mathcal{L}}$ and $\mathcal{Y}_{\mathcal{R}} \subset \tilde{\mathcal{Y}}_{\mathcal{R}}$. The ends of the tubes containing the rest points $P_{2}$ and $P_{3}$ are denoted by $N_{\mathcal{L}}^{-}$and $N_{\mathcal{R}}^{+}$, respectively, and their projections in $\left(u_{1}, v_{1}\right)$ space are denoted by $\mathcal{Y}_{\mathcal{L}}^{-}$and $\mathcal{Y}_{\mathcal{R}}^{+}$ and appear as the shaded regions in Figure 8. The monotonicity of $u_{1}$ ensures that 

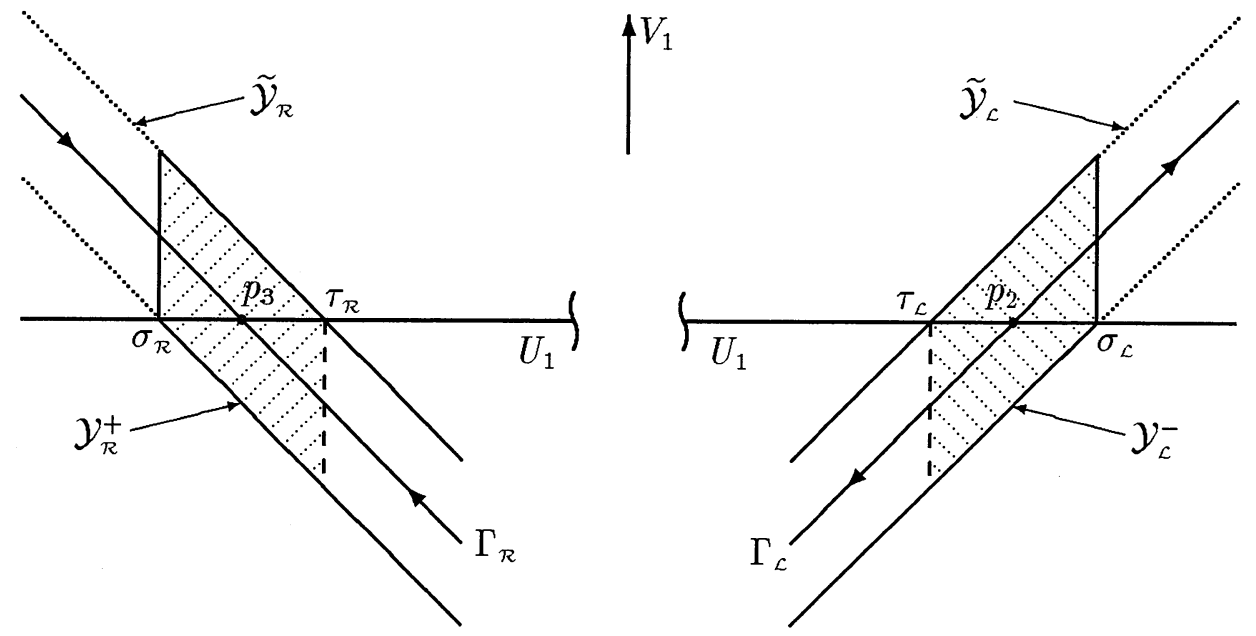

FIGURE 8. $\mathcal{Y}_{\mathcal{L}}^{-}, \mathcal{Y}_{\mathcal{R}}^{+}$: the slow tubes near the rest points.

solutions in $S\left(N_{1} ; \varepsilon\right)$ end up in $N_{\mathcal{L}}^{-}$as $\xi \rightarrow-\infty$ and in $N_{\mathcal{R}}^{+}$as $\xi \rightarrow+\infty$. The neighborhoods $N_{\mathcal{L}}^{-}$and $N_{\mathcal{R}}^{+}$will be chosen small enough to isolate the rest points $P_{2}$ and $P_{3}$ for all sufficiently small $\varepsilon>0$.

3.4.4. The neighborhood $N_{0}(\delta)$. Let $\mathcal{Y}_{0}$ be a square in $\left(u_{1}, v_{1}\right)$ space centered at $(\alpha, 0)$, with sides of length $2 \delta_{0}$, and define $N_{0}(\delta)=\mathcal{Y}_{0}\left(\delta_{0}\right) \times \mathcal{Z}_{\mathrm{f}}$. Here $\mathcal{Z}_{\mathrm{f}}$ is the isolating region defined in section 3.4.2, relative to the fast reduced flow with $u_{1}=\alpha$. For the flow at $\mu=0$ in (3.4), we have $\alpha=1$ and a four-dimensional invariant manifold $\mathcal{M}_{0}$ where the flow in $\mathcal{M}_{0}$ is the fast reduced flow with $u_{1}=1$. Recall that $S_{0}(N ; \varepsilon)$ denotes the maximal invariant set in $N \subset \mathbb{R}^{6}$ with respect to the flow in $(3.4)$ and $S_{\mathrm{f}}(\mathcal{Z} ; 1)$ denotes the maximal invariant set in $\mathcal{Z} \subset \mathbb{R}^{4}$ relative to the fast reduced flow with $u_{1}=1$. The following lemma shows that for $\delta_{0}$ sufficiently small, $\mathcal{M}_{0}$ contains the maximal invariant set of $N_{0}(\delta)$, relative to the flow in (3.4).

Lemma 3.4. Let $\mathcal{Z}$ be any compact set in $\mathbb{R}^{4}$ and $S_{f}(\mathcal{Z} ; 1)$ the maximal invariant set in $\mathcal{Z}$ relative to the fast reduced flow with $u_{1}=1$. Then for all $\delta_{0}<1 / 2$ and all $\varepsilon \in(0,1]$, the total invariant set of $\mathcal{Y}_{0}\left(\delta_{0}\right) \times \mathcal{Z}$ relative to (3.4) is given by,

$$
S_{0}\left(\mathcal{Y}_{0} \times \mathcal{Z} ; \varepsilon\right)=\{(1,0)\} \times S_{f}(\mathcal{Z} ; 1) .
$$

Proof. Let $\varphi(\xi)$ be a solution in $S_{0}\left(\mathcal{Y}_{0} \times \mathcal{Z} ; \varepsilon\right)$. By the invariance of the manifold $\mathcal{M}_{0}$ it is clear that

$$
S_{0}\left(\mathcal{Y}_{0} \times \mathcal{Z} ; \varepsilon\right) \cap \mathcal{M}_{0}=\{(1,0)\} \times S_{\mathrm{f}}(\mathcal{Z} ; 1),
$$

and if $\left(u_{1}, v_{1}\right)=(1,0)$ at any point on the solution $\varphi$, then $\varphi(\xi)$ must lie in $\mathcal{M}_{0}$ for all $\xi$. Therefore, fix $\delta_{0}>0$ and assume there is a curve $\varphi(\xi) \in S_{0}\left(\mathcal{Y}_{0} \times \mathcal{Z} ; \varepsilon\right)$ with $\varphi(\xi) \notin \mathcal{M}_{0}$ for all $\xi$. There is a largest $\bar{\delta} \leq \delta_{0}$ such that the curve $\varphi$ touches the boundary $\partial \mathcal{Y}_{0}(\bar{\delta}) \times \mathcal{Z}$. If there is such a curve $\varphi$, the flow at this point must necessarily have an internal tangency relative to the neighborhood $\mathcal{Y}_{0}(\bar{\delta}) \times \mathcal{Z}$. The idea is to show that for $\bar{\delta}<1 / 2$, the differential equations imply that such a point 


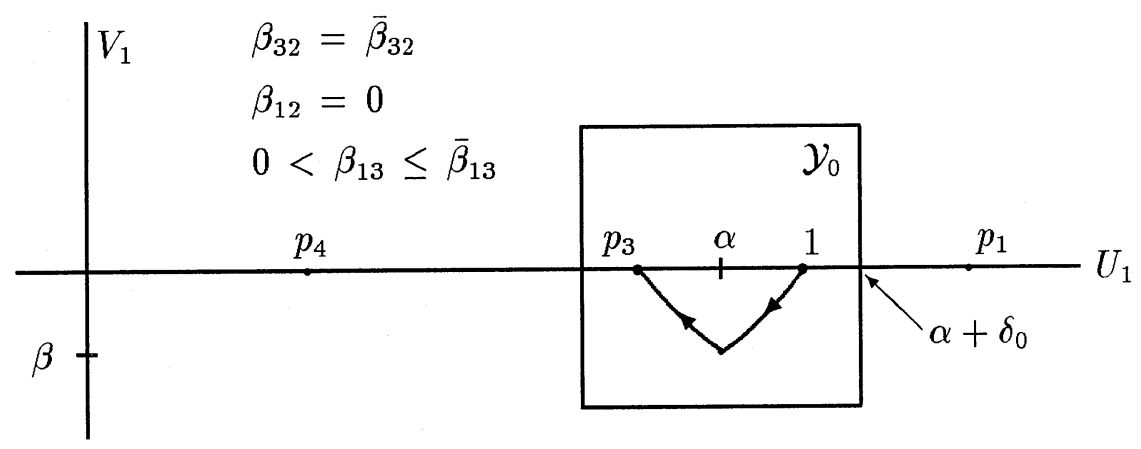

FIGURE 9. $\mathcal{Y}_{0}$ : the isolating neighborhood at $\mu=\bar{\mu}$.

has an external tangency. Then, for $\delta_{0}<1 / 2, S_{0}\left(\mathcal{Y}_{0} \times \mathcal{Z} ; \varepsilon\right)$ must lie in $\mathcal{M}_{0}$, and it follows from $(3.9)$ that $S_{0}\left(\mathcal{Y}_{0} \times \mathcal{Z} ; \varepsilon\right)=\{(1,0)\} \times S_{\mathrm{f}}(\mathcal{Z} ; 1)$.

To add some generalization to the lemma for later use, express the equation for $v_{1}^{\prime}$ as a perturbation about $u_{1}=1$ and multiply the $v_{1}$ and $\left(u_{1}-1\right)^{2}$ terms by a parameter $\lambda$ :

$$
v_{1}^{\prime}=\varepsilon\left[-\lambda \varepsilon \theta v_{1}+\left(u_{1}-1\right)+\lambda\left(u_{1}-1\right)^{2}\right] .
$$

Later, in completing the continuation, we cite the fact that this lemma holds for all $\lambda \in[0,1]$. To prove the lemma, two possibilities must be considered.

1. Suppose that $v_{1}= \pm \bar{\delta}$ and $v_{1}^{\prime}=0$. Then $v_{1}^{\prime \prime}=\varepsilon^{2} v_{1}\left[1+2 \lambda\left(u_{1}-1\right)\right]$ and for all $\bar{\delta}<1 / 2$ and $\lambda \in[0,1], \operatorname{sgn}\left(v_{1}^{\prime \prime}\right)=\operatorname{sgn}\left(v_{1}\right)$. It follows that the flow at such a point has an external tangency.

2. Suppose $u_{1}=1 \pm \bar{\delta}$ and $u_{1}^{\prime}=0$. Then $u_{1}^{\prime \prime}=\varepsilon v_{1}^{\prime}=\varepsilon^{2}( \pm \bar{\delta})(1 \pm \lambda \bar{\delta})$. If $\bar{\delta}<1$, then $u_{1}^{\prime \prime}<0$ for $u_{1}=1-\bar{\delta}$, and $u_{1}^{\prime \prime}>0$ for $u_{1}=1+\bar{\delta}$, implying that the flow at these points has an external tangency.

It follows that for the flow with $\mu=0, N_{0}^{-}=\mathcal{Y}_{0} \times \mathcal{Z}_{\mathrm{f} \mathcal{L}}$ and $N_{0}^{+}=\mathcal{Y}_{0} \times \mathcal{Z}_{\mathrm{fR}}$ isolate the rest points at $P_{2}$ and $P_{3}$, respectively, and $N_{0}=\mathcal{Y}_{0} \times \mathcal{Z}_{\mathrm{f}}$ is an isolating neighborhood in $\mathbb{R}^{6}$ with $S_{0}\left(N_{0} ; \varepsilon\right)$ consisting only of connections from $P_{2}$ to $P_{3}$.

Now consider the continuation near $\mu=0$ with $0 \leq \beta_{13} \leq \bar{\beta}_{13}, \beta_{12}=0$, and $\beta_{32}=\bar{\beta}_{32}$. This is just an $O\left(\beta_{13}\right)$ regular perturbation of the flow in (3.4). For $\bar{\beta}_{13}$ sufficiently small, the neighborhoods $N_{0}^{-}, N_{0}^{+}$, and $N_{0}$ remain isolating relative to the flow in (1.5) for all $\varepsilon \in(0,1]$. Moreover, we take $\delta_{0}$ and $\bar{\beta}_{13}$ sufficiently small that $S\left(N_{0}^{-} ; \varepsilon\right)=P_{2}, S\left(N_{0}^{+} ; \varepsilon\right)=P_{3}$, and for all $\left(u_{1}, v_{1}\right) \in \mathcal{Y}_{0}, \mathcal{Z}_{\mathrm{f}}$ is isolating for the fast reduced flow, with $S_{\mathrm{f}}\left(\mathcal{Z}_{\mathrm{f}} ; u_{1}\right)$ consisting only of connections from $\mathcal{L}\left(u_{1}\right)$ to $\mathcal{R}\left(u_{1}\right)$. In particular, we can assume that the point $g_{2}(u)=g_{3}(u)=0$ remains excised from $\mathcal{Z}_{\mathrm{f}}$ for all $y \in \mathcal{Y}_{0}$. Figure 9 shows the slow singular limit in $\left(u_{1}, v_{1}\right)$ space relative to the neighborhood $\mathcal{Y}_{0}\left(\delta_{0}\right)$ at $\mu=\bar{\mu}$, where the notation $\bar{\mu}$ is used to denote the point in the homotopy where $\beta_{32}=\bar{\beta}_{32}, \beta_{12}=0$, and $\beta_{13}=\bar{\beta}_{13}$.

3.4.5. Specifying parameters. Fix $\delta_{c}, \delta_{\mathrm{f}}$, and $\delta_{0}$ from the previous lemmas and take $0<\varepsilon \leq \bar{\varepsilon}$ satisfying Lemma 3.2. We now restrict the remaining parameters, and it will be clear that the following conditions can be satisfied by first fixing $\delta_{1}$, then $\delta_{2}$, 
and finally $\delta_{3}$. These conditions will be cited later in proving the a priori estimates and isolating properties of $N_{1}$ and $N_{0}$.

(C1) First restrict $b, \delta_{1}, \delta_{2}$, and $\delta_{3}$ so that $N_{1}(\delta) \subset N_{0}(\delta)$ at $\mu=\bar{\mu}$. For $b, \delta_{1}$, and $\delta_{2}$ sufficiently small, we have $\mathcal{Y}_{\mathrm{f}}\left(\delta_{1}\right) \cup \mathcal{Y}_{\mathcal{L}}\left(\delta_{2}\right) \cup \mathcal{Y}_{\mathcal{R}}\left(\delta_{2}\right) \subset \mathcal{Y}_{0}\left(\delta_{0}\right)$. For $\delta_{3}$ sufficiently small, $\mathcal{Z}_{\mathcal{L}}\left(\delta_{3} ; u_{1}\right) \subset \mathcal{Z}_{\mathrm{f} \mathcal{L}}$ and $\mathcal{Z}_{\mathcal{R}}\left(\delta_{3} ; u_{1}\right) \subset \mathcal{Z}_{\mathrm{f} \mathcal{R}}$ for all $\left(u_{1}, v_{1}\right) \in \mathcal{Y}_{0}$, and it follows that $N_{1} \subset N_{0}$ at $\mu=\bar{\mu}$.

(C2) We use $\delta_{1}$ to control the approximation by the fast reduced flow. In the fast scaling (1.5), the flow in the fast variables is an $O\left(\varepsilon\left|u_{1}-\alpha\right|\right)$ regular perturbation of the fast reduced flow at $u_{1}=\alpha$. Let $\xi_{\mathrm{f}}$ be the maximal exit time for all points in the boundary $\partial \mathcal{Z}_{\mathrm{f}}$ relative to this fast reduced flow. Choose $\delta_{1}$ sufficiently small so that if $z(0 ; \varepsilon) \in \partial \mathcal{Z}_{\mathrm{f}}$ and $\left|u_{1}(\xi ; \varepsilon)-\alpha\right| \leq 2 \delta_{1}$ for all $|\xi| \leq \xi_{\mathrm{f}}$, then $z(\xi ; \varepsilon)$ lies outside $\mathcal{Z}_{\mathrm{f}}$ relative to the flow in (1.5) for some $|\xi| \leq \xi_{\mathrm{f}}$.

(C3) Use $\delta_{1}$ and $\delta_{2}$ to ensure that the slow tubes $\tilde{\mathcal{Y}}_{\mathcal{L}} \backslash \mathcal{Y}_{\mathrm{f}}$ and $\tilde{\mathcal{Y}}_{\mathcal{R}} \backslash \mathcal{Y}_{\mathrm{f}}$ are uniformly bounded away from each other in $\left(u_{1}, v_{1}\right)$ space (see Figure 7$)$. For $\delta_{1}$ sufficiently small, the curves $\Gamma_{\mathcal{L}}$ and $\Gamma_{\mathcal{R}}$ intersect the boundary of $\mathcal{Y}_{\mathrm{f}}\left(\delta_{1}\right)$ along the sides $u_{1}=\alpha \pm \delta_{1}$. Now choose $\delta_{2}$ sufficiently small so that if $y$ is not in $\mathcal{Y}_{\mathrm{f}}$ then $y$ must be bounded away from at least one of the slow tubes, that is, $\operatorname{dist}\left(y, \tilde{\mathcal{Y}}_{\mathcal{L}}\right)>\delta_{2}$ or $\operatorname{dist}\left(y, \tilde{\mathcal{Y}}_{\mathcal{R}}\right)>\delta_{2}$.

(C4) Use $\delta_{1}$ and $\delta_{3}$ to minimize the type of boundary points where the slow tubes join the fast block. Make $\delta_{1}$ sufficiently small so that for $\left|u_{1}-\alpha\right| \leq 2 \delta_{1}, \mathcal{L}\left(u_{1}\right)$ is interior to $\mathcal{Z}_{\mathrm{f} \mathcal{L}}$ and $\mathcal{R}\left(u_{1}\right)$ is interior to $\mathcal{Z}_{\mathrm{f} \mathcal{R}}$. Then for $\delta_{3}$ sufficiently small and $\left(u_{1}, v_{1}\right) \in \mathcal{Y}_{\mathrm{f}}$, the set $\mathcal{Z}_{\mathcal{L}}\left(\delta_{3} ; u_{1}\right)$ lies interior to $\mathcal{Z}_{\mathrm{f} \mathcal{L}}$ and $\mathcal{Z}_{\mathcal{R}}\left(\delta_{3} ; u_{1}\right)$ remains interior to $\mathcal{Z}_{\mathrm{f} \mathcal{R}}$.

(C5) For $\delta_{2}$ and $\delta_{3}$ sufficiently small and $0<\varepsilon \leq \bar{\varepsilon}$, it follows from Lemma 3.2 that $S\left(N_{\mathcal{L}}^{-} ; \varepsilon\right)=P_{2}$ and $S\left(N_{\mathcal{R}}^{+} ; \varepsilon\right)=P_{3}$.

(C6) We use $\delta_{3}$ and $\bar{\varepsilon}$ to control the approximation to the reduced flow on the slow manifolds. Let $\mathcal{Y}_{m}$ denote a compact set in $\left(u_{1}, v_{1}\right)$ space large enough to contain the sets $\mathcal{Y}_{0}$ and $\tilde{Y}_{\mathcal{L}} \cup \mathcal{Y}_{\mathrm{f}} \cup \tilde{Y}_{\mathcal{R}}$ (see Figure 7). For the reduced flow on the left slow manifold, points in $\mathcal{Y}_{m} \backslash \tilde{\mathcal{Y}}_{\mathcal{L}}$ exit $\mathcal{Y}_{m}$ in both time directions, points in $\tilde{\mathcal{Y}}_{\mathcal{L}} \backslash \mathcal{Y}_{\mathcal{L}}$ exit $\mathcal{Y}_{m}$ in forward time, and by compactness, we can define a maximal exit time $\zeta_{\mathrm{s}}\left(\delta_{2}\right)$. In the slow scaling (1.4), the slow components $Y(\zeta ; \varepsilon)$ are uniformly approximated by the reduced flow on the left slow manifold, as long as the fast components $Z(\zeta ; \varepsilon)$ stay uniformly close to $\mathcal{L}(Y(\zeta ; \varepsilon)$ and $\varepsilon$ is sufficiently small:

$$
\dot{Y}=F(Y, \mathcal{L}(Y))+O(|Z-\mathcal{L}(Y)|)-\varepsilon \theta V_{1} .
$$

Choose $\delta_{3}$ and $\bar{\varepsilon}$ sufficiently small such that if $Y(0 ; \varepsilon) \in \mathcal{Y}_{m} \backslash \tilde{\mathcal{Y}}_{\mathcal{L}}$ and $Z(\zeta ; \varepsilon)$ stays in $\mathcal{Z}_{\mathcal{L}}\left(\delta_{3} ; U_{1}(\zeta ; \varepsilon)\right)$ for $-\zeta_{\mathrm{s}} \leq \zeta \leq 0$, then $Y(\zeta ; \varepsilon)$ exits $\mathcal{Y}_{m}$ for some $\zeta$ in the interval $\left[-\zeta_{s}, 0\right]$. Similarly, if $Z(\zeta ; \varepsilon)$ stays in $\mathcal{Z}_{\mathcal{L}}$ for $0 \leq \zeta \leq \zeta_{\mathrm{s}}$, then points in both $\mathcal{Y}_{m} \backslash \tilde{\mathcal{Y}}_{\mathcal{L}}$ and $\tilde{\mathcal{Y}}_{\mathcal{L}} \backslash \mathcal{Y}_{\mathcal{L}}$ should exit $\mathcal{Y}_{m}$ in the interval $\left[0, \zeta_{\mathrm{s}}\right]$. A similar approximation holds for the case where $Z(\zeta ; \varepsilon)$ is in the neighborhood $\mathcal{Z}_{\mathcal{R}}\left(\delta_{3} ; U_{1}(\zeta ; \varepsilon)\right)$ using approximation by the reduced flow on the right slow manifold.

3.5. A priori estimates. In this section, we establish a priori estimates for nonconstant solutions in $S\left(N_{0} ; \varepsilon\right)$ and $S\left(N_{1} ; \varepsilon\right)$, relative to the flow in (1.4) and (1.5). Lemma 3.5 shows that the total invariant sets consist only of connecting solutions from $P_{2}$ and $P_{3}$, and Lemma 3.6 shows that any such connecting solution must be uniformly close to a singular limit solution. 
Lemma 3.5. For all $\varepsilon$ sufficiently small, $S\left(N_{1} ; \varepsilon\right)$ and $S\left(N_{0} ; \varepsilon\right)$ consist only of the rest points $P_{2}$ and $P_{3}$ and connections from $P_{2}$ to $P_{3}$.

Proof. For the neighborhood $N_{1}$, the lemma follows easily from the monotonicity of $u_{1}$ and the fact that $S\left(N_{\mathcal{L}}^{-} ; \varepsilon\right)=P_{2}$ and $S\left(N_{\mathcal{R}}^{+} ; \varepsilon\right)=P_{3}$. To prove the lemma for $N_{0}$ observe that solutions entering $\mathcal{U}_{\mathrm{f} \mathcal{L}}$ in backward time remain there for all $\xi \rightarrow-\infty$ and solutions entering $\mathcal{U}_{\mathrm{f} \mathcal{R}}$ in forward time remain there for all $\xi \rightarrow+\infty$. If a solution in $S\left(N_{0} ; \varepsilon\right)$ stays outside $\left(\mathcal{U}_{\mathrm{f} \mathcal{L}} \cup \mathcal{U}_{\mathrm{f} \mathcal{R}}\right)$ for all $\xi \rightarrow-\infty$, monotonicity in $u_{2}$ and $u_{3}$ implies that the fast variables approach a limit $\left(\bar{u}_{2}, 0, \bar{u}_{3}, 0\right)$. For $\beta_{13}$ and $\varepsilon$ sufficiently small, the flow for the slow variables will be asymptotic to a saddle point at $\left(1-\beta_{13} \bar{u}_{3}, 0\right)$, and, therefore, $u(\xi ; \varepsilon)$ must be asymptotic to a rest point of the reaction flow. Two of these rest points, $P_{2}$ and $P_{3}$, lie interior to $\mathcal{U}_{\mathrm{f} \mathcal{L}} \cup \mathcal{U}_{\mathrm{f} \mathcal{R}}$ and the remaining two have been excised from $N_{0}$ for $\beta_{13} \in\left[0, \bar{\beta}_{13}\right]$. A similar argument in forward time shows that a solution in $S\left(N_{0} ; \varepsilon\right)$ cannot stay outside of $\mathcal{U}_{\mathfrak{f} \mathcal{L}} \cup \mathcal{U}_{\mathrm{f} \mathcal{R}}$ for all $\xi \rightarrow+\infty$. The lemma for $N_{0}$ follows since $\mathcal{Y}_{0} \times \mathcal{Z}_{\mathrm{f} \mathcal{L}}$ isolates $P_{2}$ and $\mathcal{Y}_{0} \times \mathcal{Z}_{\mathrm{f} \mathcal{R}}$ isolates $P_{3}$.

From the previous lemma, we can parametrize nonconstant solutions in $S\left(N_{1} ; \varepsilon\right)$ and $S\left(N_{0} ; \varepsilon\right)$ to be bounded away from both slow manifolds at $\xi=0$, for example, $u_{2}(0 ; \varepsilon)=\mathcal{L}_{2}(\alpha) / 2$. The next lemma states that any such connecting solution lies close to the slow manifolds outside a compact $\xi$-interval where the $\xi$-interval can be chosen uniformly for $\varepsilon \rightarrow 0$. The notation $\mathcal{Z}^{0}$ denotes the interior of the set $\mathcal{Z}$.

Lemma 3.6. Suppose $(x(\xi ; \varepsilon), \theta(\varepsilon))$ is a nonconstant solution in the total invariant set $S\left(N_{1} ; \varepsilon\right)$ or $S\left(N_{0} ; \varepsilon\right)$, with $u_{2}(0 ; \varepsilon)=\mathcal{L}_{2}(\alpha) / 2$. Given any $\delta_{3}>0$, there exists $\bar{\varepsilon}>0$ and $\bar{\xi}>0$ such that if $0<\varepsilon \leq \bar{\varepsilon}$, then $x(\xi ; \varepsilon)=(y(\xi ; \varepsilon), z(\xi ; \varepsilon))$ satisfies

$$
\begin{aligned}
& z(\xi ; \varepsilon) \in \mathcal{Z}_{\mathcal{L}}^{0}\left(\delta_{3} ; u_{1}(\xi ; \varepsilon)\right) \text { for all } \xi \leq-\bar{\xi}, \\
& z(\xi ; \varepsilon) \in \mathcal{Z}_{\mathcal{R}}^{0}\left(\delta_{3} ; u_{1}(\xi ; \varepsilon)\right) \text { for all } \xi \geq+\bar{\xi} .
\end{aligned}
$$

Furthermore, the wave speed $\theta(\varepsilon)$ tends to $\Theta(\alpha)$ as $\varepsilon \rightarrow 0$.

Proof. First, work in the fast scaling where solutions to (1.5) can be approximated by solutions to the fast reduced flow, uniformly on compact $\xi$-intervals as $\varepsilon \rightarrow 0$. Consider a sequence of heteroclinic orbits $\left(x\left(\hat{\xi} ; \varepsilon_{n}\right), \theta\left(\varepsilon_{n}\right)\right)$ in $S\left(N_{1} ; \varepsilon_{n}\right)\left(\operatorname{resp} . S\left(N_{0} ; \varepsilon_{n}\right)\right)$, parameterized such that $u_{2}\left(0 ; \varepsilon_{n}\right)=\mathcal{L}_{2}(\alpha) / 2$ where $\hat{\xi}$ is fixed and $\varepsilon_{n} \rightarrow 0$. Let $(\hat{x}, \hat{\theta})$ be a limit point and $x_{\mathrm{r}}(\xi)=\left(\hat{y}, z_{\mathrm{r}}(\xi)\right)$ the solution through $\hat{x}$ for the fast reduced flow parametrized by $\hat{u}_{1}$ and $\hat{\theta}$. We first show that $(\hat{x}, \hat{\theta})$ lies on a connecting solution for the fast reduced problem with $\hat{\theta}=\Theta\left(\hat{u}_{1}\right)$. By uniform approximation on the interval $[0, \hat{\xi}]$, the point $\hat{x}$ satisfies $\left|\hat{u}_{1}-\alpha\right| \leq \delta_{1}$, and it follows from (C4) that $\hat{z} \in \mathcal{Z}_{\mathrm{f}}$. If $(\hat{x}, \hat{\theta})$ does not lie on a connecting orbit for the fast reduced problem, $z_{\mathrm{r}}\left(\xi^{*}\right)$ is outside $\mathcal{Z}_{\mathrm{f}}$ at some finite $\xi^{*}$, and for $\varepsilon_{n}$ sufficiently small, $z\left(\xi^{*} ; \varepsilon_{n}\right)$ also lies outside $\mathcal{Z}_{\mathrm{f}}$ with $\left|u_{1}\left(\xi^{*} ; \varepsilon_{n}\right)-\alpha\right| \leq 2 \delta_{1}$. By condition (C4), $x\left(\xi^{*} ; \varepsilon_{n}\right)$ must lie outside $N_{1}$ as $\varepsilon_{n} \rightarrow 0$. The same result holds for the neighborhood $N_{0}=\mathcal{Y}_{0} \times \mathcal{Z}_{\mathrm{f}}$.

Let $z_{\mathrm{r}}(\xi)$ be a solution to the fast reduced problem connecting $\mathcal{L}\left(u_{1}\right)$ to $\mathcal{R}\left(u_{1}\right)$ with wave speed $\Theta\left(u_{1}\right)$ and $u_{2 \mathrm{r}}(0)=\mathcal{L}_{2}(\alpha) / 2$. Given $\delta_{3}>0$, there exists $\bar{\xi}>0$ such that

$$
\begin{aligned}
& z_{\mathrm{r}}(\xi) \in \mathcal{Z}_{\mathcal{L}}^{0}\left(\delta_{3} ; u_{1}\right) \quad \text { for all } \quad \xi \leq-\bar{\xi}, \\
& z_{\mathrm{r}}(\xi) \in \mathcal{Z}_{\mathcal{R}}^{0}\left(\delta_{3} ; u_{1}\right) \quad \text { for all } \quad \xi \geq \bar{\xi}
\end{aligned}
$$


It follows that given any fixed $\xi \geq \bar{\xi}$ and $\varepsilon$ sufficiently small, a connecting orbit $(x(\xi ; \varepsilon), \theta(\varepsilon))$ must satisfy

$$
z(-\xi ; \varepsilon) \in \mathcal{Z}_{\mathcal{L}}^{0}\left(\delta_{3} ; u_{1}(-\xi ; \varepsilon)\right) \text { and } z(\xi ; \varepsilon) \in \mathcal{Z}_{\mathcal{R}}^{0}\left(\delta_{3} ; u_{1}(\xi ; \varepsilon)\right) .
$$

It remains to show that there exists an $\bar{\varepsilon}>0$ such that if $0<\varepsilon \leq \bar{\varepsilon}$, then (3.13) holds for all $|\xi| \geq \bar{\xi}$. Suppose that the choice of $\bar{\varepsilon}$ does not hold uniformly for $\xi \rightarrow-\infty$. Then there exists a sequence $\left(x\left(\xi_{n} ; \varepsilon_{n}\right), \theta\left(\varepsilon_{n}\right)\right)$ of connections in $S\left(N_{1} ; \varepsilon\right)$ (resp. $S\left(N_{0} ; \varepsilon\right)$ ), with $\xi_{n} \rightarrow-\infty, \varepsilon_{n} \rightarrow 0$, and $z\left(\xi_{n} ; \varepsilon_{n}\right) \notin \mathcal{Z}_{\mathcal{L}}^{0}\left(\delta_{3} ; u_{1}\left(\xi_{n} ; \varepsilon_{n}\right)\right)$. Let $(\hat{x}, \hat{\theta})$ denote a limit point and $\left(x_{\mathrm{r}}(\xi), \hat{\theta}\right)$ the solution to the fast reduced flow with $x_{\mathrm{r}}(0)=\hat{x}$. From the hyperbolicity of the slow manifold, $z_{\mathrm{r}}\left(\xi^{*}\right)$ must lie outside $\mathcal{Z}_{\mathcal{L}}\left(\delta_{3} ; \hat{u}_{1}\right)$ for some finite $\xi^{*}$, as must $z\left(\xi^{*} ; \varepsilon_{n}\right)$ for $\varepsilon_{n}$ sufficiently small. It follows that the limit point $\hat{x}$ is in the fast block $N_{\mathrm{f}}=\mathcal{Y}_{\mathrm{f}} \times \mathcal{Z}_{\mathrm{f}}\left(\right.$ resp. $N_{0}=\mathcal{Y}_{0} \times \mathcal{Z}_{\mathrm{f}}$ ), and therefore, $\hat{x}$ lies on a connecting orbit to the fast reduced flow with wave speed $\hat{\theta}=\Theta\left(\hat{u}_{1}\right)$. It follows from (3.12) that this solution must lie in $\mathcal{Z}_{\mathcal{R}}^{0}\left(\delta_{3}, \hat{u}_{1}\right)$ for all $\xi \geq 2 \bar{\xi}$ and, as $\varepsilon_{n} \rightarrow 0$, the connecting solutions to (1.5) also must lie near the right slow manifold at $\xi=\xi_{n}+2 \bar{\xi}$. From the choice of parametrization and the monotonicity away from the slow manifolds, $x\left(\xi ; \varepsilon_{n}\right)$ can be near the right slow manifold only for $\xi>0$. However, as $\varepsilon_{n} \rightarrow 0, \xi_{n}+2 \bar{\xi} \rightarrow-\infty$, yielding the necessary contradiction.

Having established the estimate for the location of the connecting solutions, we now show that the wave speed tends to $\Theta(\alpha)$ as $\varepsilon \rightarrow 0$. Consider a sequence of connecting solutions $\left(x\left(0 ; \varepsilon_{n}\right) ; \theta\left(\varepsilon_{n}\right)\right)$ with $u_{2}\left(0 ; \varepsilon_{n}\right)=\mathcal{L}_{2}(\alpha) / 2$, converging to $(\hat{x}, \hat{\theta})$ with $\hat{\theta}=\Theta\left(\hat{u}_{1}\right)$. Suppose $\hat{y} \neq(\alpha,-\beta)$ and, for definiteness, assume that $\hat{y}$ does not lie on the slow singular limit on $\mathcal{M}_{\mathcal{L}}$. The solution to the reduced flow on $\mathcal{M}_{\mathcal{L}}$ with $Y(0)=\hat{Y}$ will lie outside $\mathcal{Y}_{\mathcal{L}} \cup \mathcal{Y}_{\mathrm{f}} \cup \mathcal{Y}_{\mathcal{R}}$ (resp. $\mathcal{Y}_{0}$ ) in finite time $-\zeta^{*}<0$. By the previous estimate, $Y\left(\zeta ; \varepsilon_{n}\right)$ is uniformly approximated by the reduced flow on $\mathcal{L}_{\mathcal{M}}$ on the half-line $\zeta \leq-\varepsilon_{n} \bar{\xi}$. As $\varepsilon_{n} \rightarrow 0, Y\left(-\varepsilon_{n} \bar{\xi} ; \varepsilon_{n}\right)$ tends to $\hat{Y}$, and by approximation to the slow reduced flow, $Y\left(-\varepsilon_{n} \bar{\xi}-\zeta^{*} ; \varepsilon_{n}\right)$ lies outside $\mathcal{Y}_{\mathcal{L}} \cup \mathcal{Y}_{\mathrm{f}} \cup \mathcal{Y}_{\mathcal{R}}$ (resp. $\mathcal{Y}_{0}$ ), contradicting $X\left(\zeta ; \varepsilon_{n}\right) \in S\left(N_{1} ; \varepsilon_{n}\right)$ (resp. $\left.S\left(N_{0} ; \varepsilon_{n}\right)\right)$.

Remark 1. In the following sections, we apply Lemma 3.6 as follows: if $x(\xi ; \varepsilon)$ is any nonconstant solution in $S\left(N_{1} ; \varepsilon\right)$ or $S\left(N_{0} ; \varepsilon\right)$, then $x(\xi ; \varepsilon)$ cannot stay uniformly bounded away from both of the slow manifolds for a time greater than $2 \bar{\xi}$.

3.6. Isolating property of $N_{1}$ and $N_{0}$. It follows from Lemma 3.4 that the neighborhood $N_{0}(\delta)$ is known to be isolating for $0 \leq \mu \leq \bar{\mu}$ and $0<\varepsilon \leq 1$. The following lemma establishes the necessary isolating properties for $N_{1}(\delta)$, relative to (1.4) and (1.5). The proof cites the restrictions on $\delta_{1}, \delta_{2}$, and $\delta_{3}$ as described in Section 3.4.5 and the a priori estimates from Section 3.5.

Lemma 3.7. Fix $\delta_{c}, \delta_{f}$, and $\delta_{0}$ as specified in Sections 3.4.1, 3.4.2, and 3.4.4, and fix $\delta_{1}, \delta_{2}$, and $\delta_{3}$ to satisfy conditions (C1)-(C6) as described in Section 3.4.5. Then there exists $\bar{\varepsilon}>0$ such that for $0<\varepsilon \leq \bar{\varepsilon}, N_{1}(\delta)$ is isolating relative to the flow in (1.4) and (1.5). That is, $S\left(N_{1} ; \varepsilon\right) \cap \partial N_{1}=\emptyset$, for all $\theta \in\left[\theta_{1}, \theta_{2}\right]$.

Proof. Let $x^{0}=\left(y^{0}, z^{0}\right)$ denote an arbitrary point in the boundary of the set $N_{1}$, and let $x(\xi ; \varepsilon)=(y(\xi ; \varepsilon), z(\xi ; \varepsilon))$ be the solution to (1.5) satisfying $x(0 ; \varepsilon)=x^{0}$. We must show that for all sufficiently small $\varepsilon>0$, the solution $x(\xi ; \varepsilon)$ exits $N_{1}$ in at least one time direction.

1. $x^{0} \in \partial N_{\mathcal{L}}, x^{0} \notin N_{\mathrm{f}}$ 
There are two cases to be considered, $y^{0} \in \partial \mathcal{Y}_{\mathcal{L}}$ and $z^{0} \in \partial \mathcal{Z}_{\mathcal{L}}$.

(a) Suppose $y^{0} \in \partial \mathcal{Y}_{\mathcal{L}}\left(\delta_{2}\right)$ with $u_{1}^{0}>\alpha+\delta_{1}$. If $u_{1}^{0}=\sigma_{\mathcal{L}}$, the solution leaves immediately in forward time. If $v_{1}^{0}=\Gamma_{\mathcal{L}}\left(u_{1}^{0}\right) \pm \delta_{2}$, then for $\xi<0, z(\xi ; \varepsilon) \in$ $\mathcal{Z}_{\mathcal{L}}\left(\delta_{3} ; u_{1}\right)$ as long as $x(\xi ; \varepsilon)$ stays in $N_{1}$. Using the approximation by the slow reduced flow on $\mathcal{M}_{\mathcal{L}}$, it follows that $X(\zeta ; \varepsilon)$ exits $N_{1}$ in finite negative time.

(b) Now suppose $z^{0} \in \partial \mathcal{Z}_{\mathcal{L}}\left(\delta_{3}, u_{1}^{0}\right)$. From the estimate in Lemma 3.6, $z(\xi ; \varepsilon)$ must stay in $\mathcal{Z}_{\mathcal{R}}\left(\delta_{3}, u_{1}(\xi ; \varepsilon)\right)$ for all $\xi \geq 2 \bar{\xi}$. Because $y^{0} \in \mathcal{Y}_{\mathcal{L}} \backslash \mathcal{Y}_{\mathrm{f}}$, it follows from (C3) that $y(2 \bar{\xi} ; \varepsilon) \notin \tilde{\mathcal{Y}}_{\mathcal{R}}$ for all $\varepsilon$ sufficiently small. From the approximation by the slow reduced flow on $\mathcal{M}_{\mathcal{R}}, X(\zeta ; \varepsilon)$ exits $N_{1}$ in some time $\zeta \leq \varepsilon 2 \bar{\xi}+\zeta_{\mathrm{s}}$.

2. $x^{0} \in \partial N_{\mathcal{R}}, x^{0} \notin N_{\mathrm{f}}$

The argument is similar to part 1 , with a time reversal.

3. $x^{0} \in \partial N_{\mathrm{f}}, x^{0} \notin\left(N_{\mathcal{L}} \cup N_{\mathcal{R}}\right)$

Again consider separately the cases $y^{0} \in \partial \mathcal{Y}_{\mathrm{f}}$ and $z^{0} \in \partial \mathcal{Z}_{\mathrm{f}}$.

(a) Suppose $y^{0} \in \partial \mathcal{Y}_{\mathrm{f}}$. If $u_{1}^{0}=\alpha \pm \delta_{1}$, then the solution $y(\xi ; \varepsilon)$ exits $\mathcal{Y}_{\mathrm{f}}$ immediately in one time direction. Because $x^{0}$ does not lie in either of the slow tubes, the solution $x(\xi ; \varepsilon)$ exits $N_{1}$ immediately. If $v_{1}^{0}=-\beta \pm b$, then we can use the approximation by the slow reduced flow. The parameters $b, \delta_{1}$, and $\delta_{2}$ have been chosen such that $y^{0}$ is bounded away from both slow tubes (see Figure 7). If $x^{0}$ is in $S\left(N_{1} ; \varepsilon\right)$, the estimate in Lemma 3.6 implies that at least one of the following statements will be true:

$$
\begin{aligned}
& \text { (i) } z(\xi ; \varepsilon) \in \mathcal{Z}_{\mathcal{R}}\left(\delta_{3} ; u_{1}(\xi ; \varepsilon)\right), \quad \text { for all } \xi \geq \bar{\xi} \text {, } \\
& \text { (ii) } z(\xi ; \varepsilon) \in \mathcal{Z}_{\mathcal{L}}\left(\delta_{3} ; u_{1}(\xi ; \varepsilon)\right), \quad \text { for all } \xi \leq-\bar{\xi} \text {. }
\end{aligned}
$$

In either case, we can choose $\varepsilon$ sufficiently small so that $y( \pm \bar{\xi} ; \varepsilon)$ lies outside both $\tilde{\mathcal{Y}}_{\mathcal{L}}$ and $\tilde{\mathcal{Y}}_{\mathcal{R}}$. Using approximation by the slow reduced flow, the solution $X(\zeta ; \varepsilon)$ must exit $N_{1}$ in some uniform time $\zeta_{\mathrm{s}}$ contradicting $x^{0} \in S\left(N_{1} ; \varepsilon\right)$.

(b) Suppose $z^{0} \in \partial \mathcal{Z}_{\mathrm{f}}$. Choose $\varepsilon$ sufficiently small so that $\left|u_{1}(\xi ; \varepsilon)-\alpha\right| \leq 2 \delta_{1}$ for all $|\xi| \leq \xi_{\mathrm{f}}$, where $\xi_{\mathrm{f}}$ is the maximal exit time for points in $\partial \mathcal{Z}_{\mathrm{f}}$ relative to the fast reduced flow. From approximation by the fast reduced flow, $z(\xi ; \varepsilon)$ also exits $\mathcal{Z}_{\mathrm{f}}$ in time $|\xi| \leq \xi_{\mathrm{f}}$. By condition (C4), we have $\mathcal{Z}_{\mathcal{L}}\left(\delta_{3}, u_{1}(\xi ; \varepsilon)\right) \subset \mathcal{Z}_{\mathrm{f}}$ and $\mathcal{Z}_{\mathcal{R}}\left(\delta_{3}, u_{1}\right) \subset \mathcal{Z}_{\mathrm{f}}$ for all $|\xi| \leq \xi_{\mathrm{f}}$. It follows that when $z(\xi ; \varepsilon)$ exits $\mathcal{Z}_{\mathrm{f}}$, the solution $x(\xi ; \varepsilon)$ must lie outside the slow tubes and hence, outside $N_{1}$.

4. $x^{0} \in \partial N_{\mathcal{L}} \cap \partial N_{\mathrm{f}}$

From condition (C4) all such points must have $y^{0} \in \partial \mathcal{Y}_{\mathrm{f}}$ since for $\left(u_{1}, v_{1}\right) \in \mathcal{Y}_{\mathrm{f}}$, $\partial \mathcal{Z}_{\mathcal{L}}\left(\delta_{3} ; u_{1}\right) \cap \partial \mathcal{Z}_{\mathrm{f}}=\emptyset$. That leaves just two cases to consider.

(a) Suppose $y^{0} \in \partial \mathcal{Y}_{\mathrm{f}} \cap \partial \mathcal{Y}_{\mathcal{L}}$. This is the same as part 1-a with $v_{1}^{0}=\Gamma_{\mathcal{L}}\left(u_{1}^{0}\right) \pm \delta_{2}$, and the solution exits $N_{1}$ in backward time.

(b) Suppose $y^{0} \in \partial \mathcal{Y}_{\mathrm{f}} \cap \mathcal{Y}_{\mathcal{L}}$ and $z^{0} \in \partial \mathcal{Z}_{\mathcal{L}}\left(\delta_{3} ; u_{1}^{0}\right)$. Because $y^{0}$ is bounded away from $\tilde{\mathcal{Y}}_{\mathcal{R}}$, the argument proceeds as in part $1(\mathrm{~b})$.

5. $x^{0} \in \partial N_{\mathcal{R}} \cap \partial N_{\mathrm{f}}$

The argument is similar to part 4 , with a time reversal. 
3.7. Continuation from $N_{1}$ to $N_{0}$. For $0 \leq \mu \leq \bar{\mu}$, the connection triples associated with $N_{0}$ are related by continuation, and similarly, for $\bar{\mu} \leq \mu \leq 1$, the connection triples associated with $N_{1}$ are related by continuation. It remains to be shown that for $\mu=\bar{\mu}$, the sets $N_{0}$ and $N_{1}$ determine the same connection triple.

Lemma 3.8. Given $\mu=\bar{\mu}$ as specified following Lemma 3.4, there exists $\bar{\varepsilon}>0$ such that for all $\varepsilon$ satisfying $0<\varepsilon \leq \bar{\varepsilon}, S_{\bar{\mu}}\left(N_{0} ; \varepsilon\right)=S_{\bar{\mu}}\left(N_{1} ; \varepsilon\right)$.

Proof. From condition (C1), we have $N_{1} \subset N_{0}$, and hence, $S\left(N_{1} ; \varepsilon\right) \subset S\left(N_{0} ; \varepsilon\right)$ for all $\varepsilon>0$. To get the inclusion in the other direction, we must show that for points $x^{0} \in N_{0} \backslash N_{1}$, the solution through $x^{0}$ exits $N_{0}$ in at least one time direction. First observe that because $N_{0}=\mathcal{Y}_{0} \times \mathcal{Z}_{\mathrm{f}}$, it follows that $(y, z) \in N_{0} \backslash N_{1}$ implies $y \in \mathcal{Y}_{0} \backslash \mathcal{Y}_{\mathrm{f}}$. Again we let $x(\xi ; \varepsilon)=(y(\xi ; \varepsilon), z(\xi ; \varepsilon))$ denote the solution to (1.5) satisfying $x(0 ; \varepsilon)=$ $x^{0}$. We assume $x^{0} \in S\left(N_{0} ; \varepsilon\right)$ and show that this leads to a contradiction. There are several cases to consider depending on the location of $y^{0} \in \mathcal{Y}_{0} \backslash \mathcal{Y}_{\mathrm{f}}$.

1. $y^{0} \in \mathcal{Y}_{0} \backslash\left(\tilde{\mathcal{Y}}_{\mathcal{L}} \cup \mathcal{Y}_{\mathrm{f}} \cup \tilde{\mathcal{Y}}_{\mathcal{R}}\right)$

From condition (C3), $y^{0}$ be must bounded away from at least one of the slow tubes $\tilde{\mathcal{Y}}_{\mathcal{L}}$ or $\tilde{\mathcal{Y}}_{\mathcal{R}}$. For definiteness, suppose $\operatorname{dist}\left(y^{0}, \tilde{\mathcal{Y}}_{\mathcal{L}}\right)>\delta_{2}$. From the estimate in Lemma 3.6, at least one of the following will be true:

$$
\begin{aligned}
& \text { (i) } z(\xi ; \varepsilon) \in \mathcal{Z}_{\mathcal{L}}\left(\delta_{3} ; u_{1}(\xi ; \varepsilon)\right), \quad \text { for all } \xi \leq-2 \bar{\xi} \text {, } \\
& \text { (ii) } z(\xi ; \varepsilon) \in \mathcal{Z}_{\mathcal{R}}\left(\delta_{3} ; u_{1}(\xi ; \varepsilon)\right), \quad \text { for all } \xi \geq 0 \text {. }
\end{aligned}
$$

If (i) is true, then $y(-2 \bar{\xi} ; \varepsilon) \notin \tilde{\mathcal{Y}}_{\mathcal{L}}$ for all sufficiently small $\varepsilon>0$, and the solution exits $N_{0}$ in backwards time by approximation to the slow reduced flow on $\mathcal{M}_{\mathcal{L}}$, contradicting $x^{0} \in S\left(N_{0} ; \varepsilon\right)$. If (ii) is true, then the solution exits $N_{0}$ in forward time by approximation to the slow reduced flow on $\mathcal{M}_{\mathcal{R}}$. A similar argument holds for the case where $\operatorname{dist}\left(y^{0}, \tilde{\mathcal{Y}}_{\mathcal{R}}\right)>\delta_{2}$.

2. $y^{0} \in \mathcal{Y}_{\mathcal{L}} \backslash \mathcal{Y}_{\mathrm{f}}$

Since $\left(y^{0}, z^{0}\right)$ lies outside $N_{1}$, it follows that $z^{0} \notin \mathcal{Z}_{\mathcal{L}}\left(\delta_{3} ; u_{1}^{0}\right)$, and therefore, $z(\xi ; \varepsilon)$ must be in $\mathcal{Z}_{\mathcal{R}}\left(\delta_{3}, u_{1}(\xi ; \varepsilon)\right)$ for all $\xi \geq 2 \bar{\xi}$. For all $\varepsilon$ sufficiently small, $y(2 \bar{\xi} ; \varepsilon)$ lies outside $\tilde{\mathcal{Y}}_{\mathcal{R}}$, and from the approximation by the slow reduced flow on $\mathcal{M}_{\mathcal{R}}$, this solution must exit $N_{0}$ in time $\zeta \leq \varepsilon 2 \bar{\xi}+\zeta$ s.

3. $y^{0} \in \mathcal{Y}_{\mathcal{R}} \backslash \mathcal{Y}_{\mathrm{f}}$

The argument is similar to part 2, with a time reversal.

4. $y^{0} \in \tilde{\mathcal{Y}}_{\mathcal{L}} \backslash\left(\mathcal{Y}_{\mathcal{L}} \cup \mathcal{Y}_{\mathrm{f}}\right)$

Applying the estimate in Lemma 3.6, at least one of the following will be true,

$$
\begin{aligned}
\text { (i) } \quad z(\xi ; \varepsilon) \in \mathcal{Z}_{\mathcal{L}}\left(\delta_{3} ; u_{1}(\xi ; \varepsilon)\right), & \text { for all } \xi \leq 0, \\
\text { (ii) } \quad z(\xi ; \varepsilon) \in \mathcal{Z}_{\mathcal{R}}\left(\delta_{3} ; u_{1}(\xi ; \varepsilon)\right), & \text { for all } \xi \geq 2 \bar{\xi} .
\end{aligned}
$$

If (ii) is true, then $y(2 \bar{\xi} ; \varepsilon) \notin \tilde{\mathcal{Y}}_{\mathcal{R}}$ for all sufficiently small $\varepsilon$, and the solution exits $N_{0}$ in forward time by approximation to the slow reduced flow on $\mathcal{M}_{\mathcal{R}}$. If (i) is true, let $\zeta_{*} \geq 0$ denote the first time at which $Z\left(\zeta_{*} ; \varepsilon\right) \in \partial \mathcal{Z}_{\mathcal{L}}$. Because the flow is well approximated by the slow reduced flow on $\mathcal{M}_{\mathcal{L}}$ for $0 \leq \zeta \leq \zeta_{*}$, we can assume $Y\left(\zeta_{*} ; \varepsilon\right) \notin \mathcal{Y}_{\mathcal{L}} \cup \mathcal{Y}_{\mathrm{f}}$. If $Y\left(\zeta_{*} ; \varepsilon\right) \notin \tilde{\mathcal{Y}}_{\mathcal{L}}$, then the solution must exit $N_{0}$ at some time $\zeta \leq \zeta_{*}$ because of the approximation by the slow reduced flow on $\mathcal{M}_{\mathcal{L}}$ for $\zeta \leq \zeta_{*}$. If $Y\left(\zeta_{*} ; \varepsilon\right) \in \tilde{\mathcal{Y}}_{\mathcal{L}}$, then the solution must satisfy $Z(\zeta ; \varepsilon) \in \mathcal{Z}_{\mathcal{R}}\left(\delta_{3} ; U_{1}(\zeta ; \varepsilon)\right)$ for all $\zeta \geq$ 
$\zeta_{*}+\varepsilon 2 \bar{\xi}$ with $Y\left(\zeta_{*}+\varepsilon 2 \bar{\xi} ; \varepsilon\right) \notin \tilde{\mathcal{Y}}_{\mathcal{R}}$. From the approximation by the slow reduced flow on $\mathcal{M}_{\mathcal{R}}$ for $\zeta \geq \zeta_{*}+\varepsilon 2 \bar{\xi}, X(\zeta ; \varepsilon)$ exits $N_{0}$ in forward time, contradicting $x^{0} \in S\left(N_{0} ; \varepsilon\right)$. 5. $y^{0} \in \tilde{\mathcal{Y}}_{\mathcal{R}} \backslash\left(\mathcal{Y}_{\mathcal{R}} \cup \mathcal{Y}_{\mathrm{f}}\right)$

The argument is similar to part 4 , with a time reversal.

3.8. Computing the index. We have shown that $N_{1}$ determines a connection triple $\left(S_{1}^{\prime}, S_{1}^{\prime \prime}, S_{1}\right)$ relative to the flow at $\mu=1, N_{0}$ determines a connection triple $\left(S_{0}^{\prime}, S_{0}^{\prime \prime}, S_{0}\right)$ relative to the flow at $\mu=0$, and their indices are the same. Introduce a continuation parameter $\lambda \in[0,1]$ into the flow at $\mu=0$, with $\lambda=1$ corresponding to the flow in (3.4):

$$
\begin{aligned}
u_{1}^{\prime} & =\varepsilon v_{1} \\
v_{1}^{\prime} & =\varepsilon\left[-\lambda \varepsilon \theta v_{1}+\left(u_{1}-1\right)+\lambda\left(u_{1}-1\right)^{2}\right] \\
u_{2}^{\prime} & =v_{2} \\
v_{2}^{\prime} & =-\theta v_{2}-r_{2} u_{2} g_{2}\left(1, u_{2}, u_{3}\right)+\lambda O\left(\varepsilon\left|u_{1}-1\right|\right) \\
u_{3}^{\prime} & =v_{3} \\
v_{3}^{\prime} & =d^{-1}\left[-\theta v_{3}-r_{3} u_{3} g_{3}\left(1, u_{2}, u_{3}\right)+\lambda O\left(\varepsilon\left|u_{1}-1\right|\right) .\right.
\end{aligned}
$$

It was shown in Lemma 3.4 that $N_{0}$ is isolating relative to the flow in (3.14) for all $\lambda \in[0,1]$. At $\lambda=0$, the system decouples into a product flow, a constant coefficient linear flow on $\mathbb{R}^{2}$ and a parametrized flow on $\mathbb{R}^{4} \times\left[\theta_{1}, \theta_{2}\right]$. Consider the triple of invariant sets in $\mathcal{R}^{4}, \tilde{S}_{\theta}^{\prime}=\mathcal{L}(1), \tilde{S}_{\theta}^{\prime \prime}=\mathcal{R}(1)$, and $\tilde{S}_{\theta}=S_{\mathrm{f}}\left(\mathcal{Z}_{\mathrm{f}} ; 1\right)$. These define a connection triple for the flow on $\mathbb{R}^{4} \times\left[\theta_{1}, \theta_{2}\right]$ with index $\bar{h}\left(\tilde{S}^{\prime}, \tilde{S}^{\prime \prime}, \tilde{S}\right)=\overline{0}$, as shown in [5]. For the flow in the slow variables, $\mathcal{Y}_{0}$ isolates the saddle point at $(1,0)$ with index $\Sigma^{1}$. Applying the formula in (3.3) to compute the index,

$$
\bar{h}\left(S_{1}^{\prime}, S_{1}^{\prime \prime}, S_{1}\right)=\Sigma^{1} \wedge \bar{h}\left(\tilde{S}^{\prime}, \tilde{S}^{\prime \prime}, \tilde{S}\right)=\Sigma^{1} \wedge \overline{0}=\overline{0} .
$$

The indices for the isolated rest points are $h\left(P_{2}\right)=h\left(P_{3}\right)=\Sigma^{3}$, and since

$$
\left(h\left(S_{1}^{\prime}\right) \wedge \Sigma^{1}\right) \vee h\left(S_{1}^{\prime \prime}\right)=\Sigma^{4} \vee \Sigma^{3} \neq \overline{0}=\bar{h}\left(S_{1}^{\prime}, S_{1}^{\prime \prime}, S_{1}\right),
$$

it follows from (3.1) that $S_{1}=S_{1}\left(N_{1} \times\left[\theta_{1}, \theta_{2}\right]\right) \neq S_{1}^{\prime} \cup S_{1}^{\prime \prime}$. By the a priori estimate in Lemma 3.5, there must exist a connection from $P_{2}$ to $P_{3}$ for some wave speed $\theta(\varepsilon) \in\left(\theta_{1}, \theta_{2}\right)$.

Acknowledgements. This research was supported in part by the National Science Foundation grants DMS-8922384 and DMS-9300848-001 and by the Office of Naval Research contract N00014-92-J-1481. The author thanks Dr. Robert Gardner and Dr. Christopher Jones for their valuable assistance and encouragement.

\section{References}

1. A. Bose, Existence and stability of travelling waves for coupled nerve axon equations, $\mathrm{PhD}$ thesis, Brown University, 1993.

2. J. Carr, Applications of Centre Manifold Theory, Springer-Verlag, 1981.

3. C. Conley, Isolated Invariant Sets and the Morse Index, CBMS Regional Conference Series in Mathematics 38, American Mathematical Society, Providence, R.I., 1978.

4. (Eds. Nitecki and C. Robinson), Lecture Notes in Mathematics 819, Springer-Verlag, 1981, pp. 65-89. 
5. C. Conley and R. Gardner, An application of the generalized Morse index to travelling wave solutions of a competitive reaction-diffusion model, Indiana University Mathematics Journal 33 (1984), 319-343.

6. N. Fenichel, Persistence and smoothness of invariant manifolds for flows, Indiana Univ. Math J. 21 (1971), 193-226.

7. P. Fife, Mathematical Aspects of Reacting and Diffusing Systems, Lecture Notes in Biomathematics 28, Springer-Verlag, 1979.

8. R. Gardner, Existence and stability of travelling wave solutions of competition models: $A$ degree theoretic approach, J. Diff. Eqns. 44 (1980), 343-364.

9. __ Existence of travelling wave solutions of predator-prey systems via the connection index, SIAM Journal of Applied Mathematics 44 (1984), 56-78.

10. - Topological methods for the study of travelling wave solutions of reaction-diffusion systems, In: Reaction-Diffusion Equations, (Eds. K.J. Brown and A.A. Lacey), pp. 173-198, 1990.

11. R. Gardner and C. K. R. T. Jones, Stability of travelling wave solutions of diffusive predator-prey systems, Transactions of the American Mathematical Society 327 (1991), 465-524.

12. R. Gardner and J. Smoller, The existence of periodic travelling waves for singularly perturbed predator-prey equations via the Conley index, J. Diff. Eqns. 47 (1983), 133-161.

13. C. K. R. T. Jones, Geometric singular perturbation theory, In: Dynamical Systems, (Ed. R. Johnson), Lecture Notes in Mathematics 1609, Springer-Verlag, 1995a, pp. 44-118.

14. C. K. R. T. Jones and N. Kopell, Tracking invariant manifolds with differential forms in singularly perturbed systems, J. Diff. Eqns. 108 (1995), 64-88.

15. K. Kishimoto, The diffusive Lotka-Volterra system with three species can have a stable nonconstant equilibrium solution, J. Math. Biology 16 (1982), 103-112.

16. P. Miller, Stability of travelling waves for a three-component reaction-diffusion system, $\mathrm{PhD}$ thesis, University of Massachusetts, Amherst, 1994.

17. M. Mimura and P. Fife, A 3-component system of competition and diffusion, Hiroshima Math. J. 16 (1986), 189-207.

18. K. Mischaikow, Travelling waves for a cooperative and a competitive-cooperative system, In: Viscous Profiles and Numerical Methods for Shock Waves, (Ed. M. Shearer) SIAM, 1991 pp. $125-141$.

19. K. Mischaikow and V. Hutson, Travelling waves for mutualist species, Technical Report 38, Center for Dynamical Systems and Nonlinear Studies, Georgia Institute of Technology, 1990.

20. J. Murray, Mathematical Biology, Springer-Verlag, 1990.

21. Y. Nishiura and M. Mimura, Layer oscillations in reaction-diffusion systems, SIAM J. Appl. Math. 49 (1989), 481-514.

22. J. Smoller, Shock Waves and Reaction-Diffusion Equations, Springer Verlag, New York, 1983.

23. A. I. Vol'pert and V. A. Vol'pert, Applications of the rotation theory of vector fields to the study of wave solutions of parabolic equations, Transactions of the Moscow Mathematical Society (1990); English transl. in Trans. Amer. Math. Soc. (1991).

Department of Mathematics, University of Massachusetts, Amherst, Massachusetts 01003, U.S.A.

Current address: Department of Mathematical Sciences, Stevens Institute of Technology, Castle Point on Hudson, Hoboken, NJ 07030, U.S.A.

E-mail: pmiller@stephens-tech.edu 